\title{
Barbados: Statistical Appendix
}

This Statistical Appendix for Barbados was prepared by a staff team of the International Monetary Fund as background documentation for the periodic consultation with the member country. It is based on the information available at the time it was completed on April 16, 2004. The views expressed in this document are those of the staff team and do not necessarily reflect the views of the government of Barbados or the Executive Board of the IMF.

The policy of publication of staff reports and other documents by the IMF allows for the deletion of market-sensitive information.

To assist the IMF in evaluating the publication policy, reader comments are invited and may be sent by e-mail to publicationpolicy@imf.org.

Copies of this report are available to the public from

International Monetary Fund $\bullet$ Publication Services

700 19th Street, N.W. • Washington, D.C. 20431

Telephone: (202) 6237430 • Telefax: (202) 6237201

E-mail: publications@imf.org • Internet: http://www.imf.org

Price: $\$ 15.00$ a copy

\section{International Monetary Fund} Washington, D.C. 



\section{INTERNATIONAL MONETARY FUND}

\section{BARBADOS \\ Statistical Appendix \\ Prepared by staff team consisting of Carlene Francis, Saqib Rizavi, and \\ Wendell Samuel (all WHD)}

Approved by Western Hemisphere Department

April 16, 2004

Contents

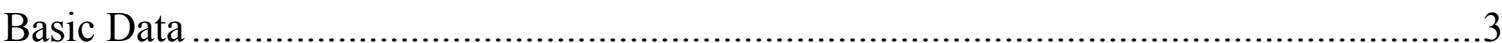

Statistical Appendix Tables

I. Real Sector

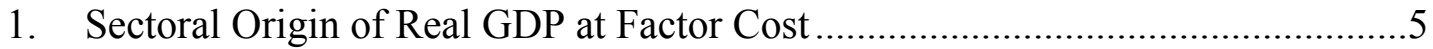

2. Index of Industrial Production .....................................................................6

3. Selected Sugar Statistics .....................................................................

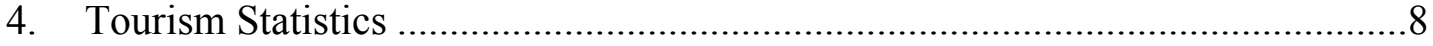

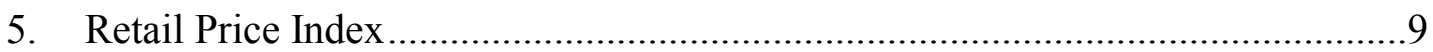

6. Selected Labor Statistics ............................................................................ 10

7. Public Service Wage Increases .................................................................... 11

II. Public Sector

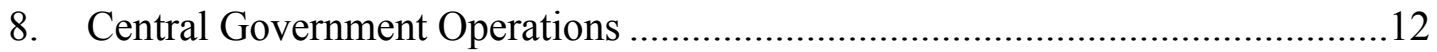

9. Central Government Revenue and Grants .......................................................... 13

10. Central Government Transfers......................................................................... 14

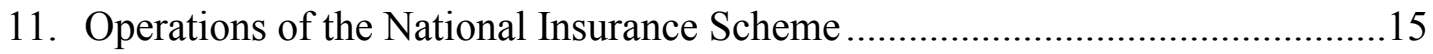

12. National Insurance Scheme: Investments and Cash Balances ..........................16

13. Public Sector Financing Requirement and Debt ......................................... 17

14. Operations of Selected Nonfinancial Public Enterprises .................................. 18 
III. Financial Sector

15. Summary Accounts of the Consolidated Financial System ..............................21

16. Summary Accounts of the Consolidated Banking System................................22

17. Summary Accounts of the Consolidated Nonbank Financial Intermediaries .....24

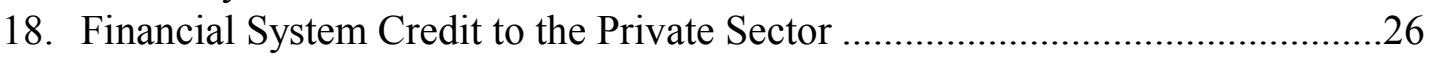

19. Liquidity Position of Commercial Banks..........................................................27

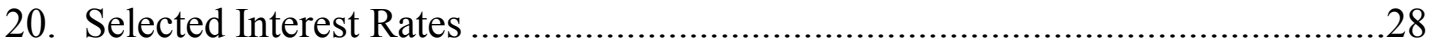

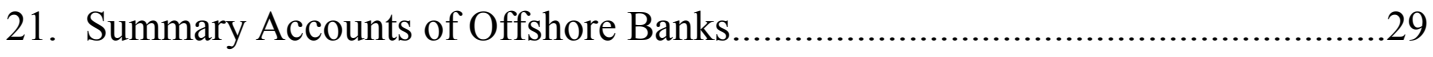

IV. External Sector

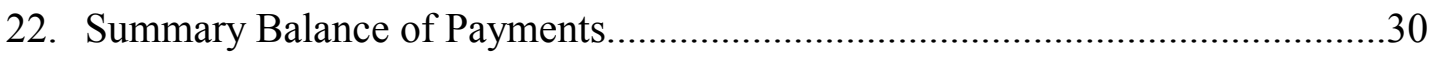

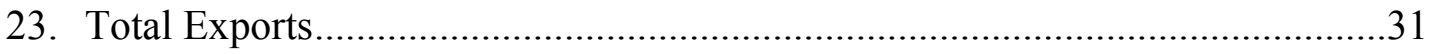

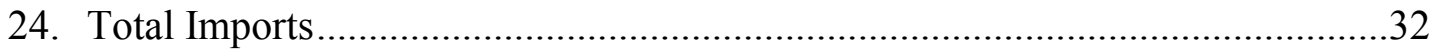

25. Services, Investment Income, and Current Transfers .......................................33

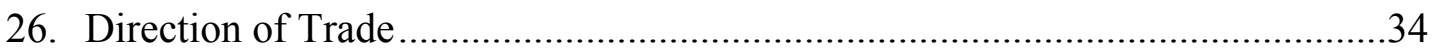

Annex

1. Summary of the Tax System as of March 31, 2004 .......................................35 


\section{Barbados: Basic Data}

I. Social and Demographic Indicators

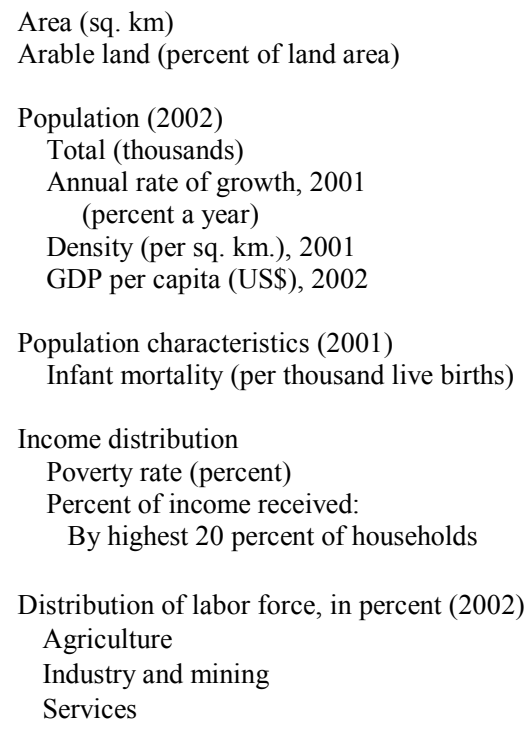

Distribution of labor force, in percent (2002) Agriculture

Industry and mining

Services

Nutrition

Calorie intake (per capita a day

Health (1994)

Population per physician $\quad 749$

Population per hospital bed

Access to electricity (1988)

Percent of population

Access to safe water (1993)

Percent of population

Education (percent) Adult literacy rate (25 and over $\quad 99$

Primary school enrollment rate

Unemployment (percent) (2002)

$\begin{array}{rlr}4.0 & \text { GDP (2003) } & \\ 18.8 & \text { Millions of Barbados dollars } & 5,391.0 \\ 77.2 & \text { Millions of U.S. dollars } & 2,695.5\end{array}$

II. Economic Indicators, 1996-2001

\begin{tabular}{llllll}
\hline & 1999 & 2000 & 2001 & 2002 & 2003
\end{tabular}

(In percent of GDP)

\section{Origin of GDP}

Agriculture and mining

Manufacturing and construction

Services

$\begin{array}{rrrrr}7.0 & 7.0 & 6.5 & 6.3 & 5.8 \\ 20.4 & 20.1 & 19.8 & 20.2 & 20.0 \\ 72.6 & 72.9 & 73.6 & 73.5 & 74.2\end{array}$

(Annual percentage changes, unless otherwise indicated)

National accounts and prices

Real GDP

Real GDP per capita

GDP deflator

Consumer price index (period average)

Consumer price index (end of period)

Unemployment rate (in percent)

Public sector (fiscal year basis) $1 /$

Government revenue

Government expenditure Of which: off-budget

Government balance

Of which: budgetary balance

Public enterprises balance (after transfers)

Nonfinancial public sector balance

Government and government guaranteed debt Of which : government debt

$\begin{array}{rr}0.5 & 2.4 \\ 0.0 & 1.9 \\ 1.7 & 1.3 \\ 1.6 & 2.4 \\ 1.6 & 2.4 \\ 10.4 & 9.4\end{array}$

(In percent of GDP)

32.1

33.4

$-1.3$

-1.3
-1.7

0.0

$-1.2$

68.1

58.5

-3.4
-3.5
2.9
2.8
2.6
9.9

$-0.4$

(1)

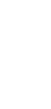




\section{Barbados: Basic Data}

\begin{tabular}{|c|c|c|c|c|c|}
\hline & 1999 & 2000 & 2001 & 2002 & 2003 \\
\hline \multicolumn{6}{|c|}{ (12-month percentage changes, unless otherwise indicated) } \\
\hline \multicolumn{6}{|l|}{ Money and credit } \\
\hline $\begin{array}{l}\text { Liabilities to private sector } \\
\text { Of which }\end{array}$ & 11.6 & 7.9 & 5.6 & 10.4 & 6.5 \\
\hline Money & 13.1 & 6.9 & 7.7 & 25.5 & 6.2 \\
\hline Quasi money & 10.8 & 8.4 & 4.6 & 2.6 & 6.6 \\
\hline $\begin{array}{l}\text { Net domestic assets of the banking system 2/ } \\
\text { Of which }\end{array}$ & 7.8 & -4.2 & -8.6 & 5.7 & -2.7 \\
\hline Credit to the public sector (net) $2 /$ & -0.4 & -4.3 & -8.1 & 12.2 & 3.6 \\
\hline Credit to the private sector $2 /$ & 11.6 & 2.1 & -0.3 & 2.4 & 0.6 \\
\hline Liabilities to private sector in percent of GDP & 59.9 & 61.9 & 65.9 & 71.9 & 73.8 \\
\hline 3-month T-bill rate $3 /$ & 6.1 & 3.9 & 2.0 & 1.5 & 0.6 \\
\hline \multicolumn{6}{|c|}{ (In millions of U.S. dollars, unless otherwise indicated) } \\
\hline \multicolumn{6}{|l|}{ Balance of payments } \\
\hline Current account & -149 & -145 & -94 & -171 & -210 \\
\hline Merchandise trade balance & -716 & -744 & -681 & -702 & -797 \\
\hline Exports & 274 & 286 & 271 & 251 & 229 \\
\hline Imports & 989 & 1,030 & 952 & 953 & 1,025 \\
\hline Services (net) & 571 & 603 & 587 & 546 & 584 \\
\hline Travel (credit) & 687 & 723 & 714 & 658 & 698 \\
\hline Investment income (net) & -71 & -82 & -93 & -102 & -96 \\
\hline Current transfers (net) & 67 & 78 & 94 & 86 & 99 \\
\hline Capital and financial account & 167 & 410 & 317 & 147 & 278 \\
\hline Long-term capital & 111 & 287 & 277 & 13 & 102 \\
\hline Public sector & 56 & 123 & 152 & 0 & -15 \\
\hline Private sector & 55 & 164 & 125 & 13 & 117 \\
\hline Overall balance & 37 & 178 & 223 & -24 & 67 \\
\hline Current account (in percent of GDP) & -6 & -6 & -4 & -6 & -8 \\
\hline Gross international reserves (millions of U.S. dollars) & 312 & 484 & 707 & 683 & 751 \\
\hline Gross international reserves (in months of imports) & 3 & 4 & 6 & 6 & 6 \\
\hline Public external debt (in millions of U.S. dollars) & 464 & 583 & 743 & 707 & 712 \\
\hline Public external debt (in percent of GDP) & 19 & 23 & 29 & 27 & 26 \\
\hline
\end{tabular}

IMF data (as of 3/31/04)

Membership status:

Intervention currency and rate

Quota

Fund holdings of Barbados dollars

(as percent of quota)

Outstanding purchases and loans

SDR department

Net cumulative allocation

Holdings

Article VIII U.S. dollar at BD\$2.0 per U.S. dollar SDR 67.50 million SDR 62.48 million 92.56 percent None

SDR 8.04 million SDR 0.01 million

Sources: Barbadian authorities; and Fund staff estimates.

1/ Fiscal year begins April 1.

2/ In relation to liabilities to the private sector at the beginning of the period.

3/ Average rate of discount (end of period). 
Table 1. Barbados: Sectoral Origin of Real GDP at Factor Cost

\begin{tabular}{|c|c|c|c|c|c|}
\hline & 1999 & 2000 & 2001 & 2002 & 2003 \\
\hline & \multicolumn{4}{|c|}{ (In millions of Barbados dollars at 1974 prices) } & \\
\hline GDP & 975 & 999 & 964 & 960 & 981 \\
\hline Primary sector & 68 & 70 & 63 & 60 & 57 \\
\hline Sugar & 23 & 25 & 21 & 19 & 16 \\
\hline Other agriculture and fishing & 36 & 36 & 34 & 34 & 34 \\
\hline Mining and quarrying & 10 & 9 & 8 & 7 & 7 \\
\hline Secondary sector & 199 & 200 & 191 & 194 & 196 \\
\hline Manufacturing & 89 & 88 & 81 & 81 & 81 \\
\hline Construction & 72 & 74 & 71 & 73 & 75 \\
\hline Electricity, gas, and water & 38 & 38 & 39 & 40 & 41 \\
\hline Services & 708 & 728 & 710 & 705 & 728 \\
\hline Wholesale and retail trade & 187 & 190 & 184 & 185 & 191 \\
\hline Tourism & 144 & 157 & 148 & 144 & 154 \\
\hline Transport and communications & 82 & 83 & 83 & 81 & 82 \\
\hline Business and general services & 164 & 165 & 163 & 160 & 163 \\
\hline \multirow[t]{2}{*}{ Government services } & 131 & 134 & 132 & 136 & 138 \\
\hline & \multicolumn{4}{|c|}{ (In percent of GDP) } & \\
\hline GDP & 100.0 & 100.0 & 100.0 & 100.0 & 100.0 \\
\hline Primary sector & 7.0 & 7.0 & 6.5 & 6.3 & 5.8 \\
\hline Sugar & 2.3 & 2.5 & 2.2 & 2.0 & 1.6 \\
\hline Other agriculture and fishing & 3.7 & 3.6 & 3.5 & 3.5 & 3.5 \\
\hline Mining and quarrying & 1.0 & 0.9 & 0.8 & 0.7 & 0.7 \\
\hline Secondary sector & 20.4 & 20.1 & 19.8 & 20.2 & 20.0 \\
\hline Manufacturing & 9.1 & 8.8 & 8.4 & 8.5 & 8.2 \\
\hline Construction & 7.4 & 7.4 & 7.4 & 7.6 & 7.7 \\
\hline Electricity, gas, and water & 3.9 & 3.8 & 4.1 & 4.1 & 4.1 \\
\hline Services & 72.6 & 72.9 & 73.6 & 73.5 & 74.2 \\
\hline Wholesale and retail trade & 19.2 & 19.0 & 19.0 & 19.3 & 19.5 \\
\hline Tourism & 14.8 & 15.7 & 15.3 & 15.0 & 15.7 \\
\hline Transport and communications & 8.4 & 8.3 & 8.6 & 8.4 & 8.3 \\
\hline Business and general services & 16.9 & 16.5 & 16.9 & 16.6 & 16.6 \\
\hline \multirow[t]{2}{*}{ Government services } & 13.4 & 13.5 & 13.7 & 14.2 & 14.1 \\
\hline & \multicolumn{4}{|c|}{ (Annual percentage changes) } & \\
\hline GDP & 0.5 & 2.4 & -3.4 & -0.4 & 2.1 \\
\hline Primary sector & 8.7 & 2.1 & -9.8 & -4.4 & -6.1 \\
\hline Sugar & 8.8 & 9.7 & -14.7 & -9.8 & -19.2 \\
\hline Other agriculture and fishing & 9.4 & 0.0 & -5.8 & -0.6 & 1.8 \\
\hline Mining and quarrying & 5.6 & -8.4 & -12.6 & -6.6 & -8.5 \\
\hline Secondary sector & 3.0 & 0.8 & -4.5 & 1.6 & 1.1 \\
\hline Manufacturing & -2.5 & -0.5 & -8.2 & 0.2 & -1.0 \\
\hline Construction & 9.7 & 2.6 & -4.4 & 3.1 & 3.0 \\
\hline Electricity, gas, and water & 4.7 & 0.0 & 3.7 & 1.8 & 1.8 \\
\hline Services & -1.1 & 2.9 & -2.5 & -0.7 & 3.2 \\
\hline Wholesale and retail trade & -7.8 & 1.3 & -3.1 & 1.0 & 3.1 \\
\hline Tourism & -0.1 & 9.2 & -5.9 & -2.8 & 7.3 \\
\hline Transport and communications & 3.7 & 0.9 & 0.6 & -3.0 & 1.4 \\
\hline Business and general services & 0.2 & 0.2 & -0.8 & -2.4 & 1.9 \\
\hline Government services & 3.8 & 2.8 & -1.6 & 3.0 & 1.3 \\
\hline
\end{tabular}

Sources: Central Bank of Barbados; and Fund staff estimates. 
Table 2. Barbados: Index of Industrial Production 1/

$(1982=100)$

\begin{tabular}{|c|c|c|c|c|c|c|}
\hline & Weights & 1999 & 2000 & 2001 & 2002 & 2003 \\
\hline Total & 100.0 & 134.6 & 125.9 & 121.1 & 124.4 & $\ldots$ \\
\hline Mining and quarrying & 5.3 & 177.4 & 154.8 & 124.9 & 119.7 & $\ldots$ \\
\hline Electricity and gas & 10.2 & 234.2 & 237.2 & 247.1 & 249.9 & $\ldots$ \\
\hline Manufacturing & 84.5 & 119.9 & 110.7 & 105.7 & 109.5 & $\ldots$ \\
\hline Food & 15.6 & 156.1 & 150.5 & 138.4 & 150.2 & $\ldots$ \\
\hline Beverages and tobacco & 9.7 & 179.5 & 154.0 & 181.5 & 175.2 & ... \\
\hline Wearing apparel & 12.2 & 7.0 & 4.2 & 1.9 & 3.5 & $\ldots$ \\
\hline Wooden furniture & 2.9 & 21.5 & 17.5 & 16.7 & 16.5 & $\ldots$ \\
\hline Chemicals & 4.6 & 110.2 & 105.7 & 73.2 & 79.4 & ... \\
\hline Petroleum products & 1.8 & $\ldots$ & $\ldots$ & $\ldots$ & $\ldots$ & $\ldots$ \\
\hline Nonmetallic mineral products & 3.4 & 155.5 & 160.1 & 147.0 & 116.9 & $\ldots$ \\
\hline Electronic products & 11.0 & 41.4 & 34.1 & 21.0 & 23.5 & $\ldots$ \\
\hline Other manufacturing & 23.3 & 185.3 & 171.9 & 166.0 & 175.8 & ... \\
\hline
\end{tabular}

Source: Central Bank of Barbados Annual Statistical Digest.

1/ End of period. 
Table 3. Barbados: Selected Sugar Statistics

\begin{tabular}{|c|c|c|c|c|c|}
\hline & 1999 & 2000 & 2001 & 2002 & 2003 \\
\hline & \multicolumn{5}{|c|}{ (Crop years: July-June) } \\
\hline Sugar production (thousands of metric tons) & 53 & 58 & 50 & 45 & 36 \\
\hline Sugarcane milled (thousands of metric tons) & 522 & 538 & 420 & 418 & 365 \\
\hline Area harvested (thousands of hectares) & 8.5 & 8.7 & 8.3 & 7.9 & 7.4 \\
\hline \multicolumn{6}{|l|}{ Average yield } \\
\hline Cane/sugar & 9.8 & 9.2 & 8.4 & 9.3 & 10.1 \\
\hline Cane/hectare harvested (metric tons) & 61 & 63 & 50 & 53 & 50 \\
\hline \multicolumn{6}{|l|}{ Quota (thousands of metric tons) } \\
\hline EC & 55 & 55 & 55 & 55 & 55 \\
\hline \multirow[t]{2}{*}{ United States } & 7.6 & 7.6 & 7.6 & 7.6 & 7.6 \\
\hline & \multicolumn{5}{|c|}{ (Calendar years) } \\
\hline Factory receipts (BDS\$ million) & 55 & 59 & 47 & 39 & 45 \\
\hline Factory receipts per metric ton (BDS\$) & 1,155 & 1,023 & 941 & 785 & 915 \\
\hline Production cost per metric ton (BDS\$) & 1,482 & 1,367 & 1,549 & 1,796 & 2,073 \\
\hline Profit or loss per metric ton (percent of cost) $3 /$ & -34.2 & -2.8 & -12.1 & -33.7 & -25.0 \\
\hline Export receipts (BDS\$ million) $1 /$ & 53 & 57 & 47 & 40 & 42 \\
\hline \multicolumn{6}{|l|}{ Exports of sugar and fancy molasses } \\
\hline (thousands of metric tons) & 48 & 58 & 49 & 40 & 34 \\
\hline Export unit value per metric ton (BDS\$) & 1,116 & 992 & 964 & 1,022 & 1,234 \\
\hline \multicolumn{6}{|l|}{ Average employment during out-of-crop season } \\
\hline Sugar estates & 429 & 411 & 428 & 366 & 358 \\
\hline Sugar factories & 301 & 306 & 361 & 272 & 336 \\
\hline
\end{tabular}

Sources: Barbados Agricultural Management Co. Ltd.; Barbados Sugar Industry Ltd.; and Ministry of Finance and Economic Affairs. 
Table 4. Barbados: Tourism Statistics

\begin{tabular}{|c|c|c|c|c|c|}
\hline & 1999 & 2000 & 2001 & 2002 & 2003 \\
\hline & \multicolumn{3}{|c|}{ (In units as indicated) } & & \\
\hline Total tourist days (thousands) $1 /$ & 4,344 & 4,510 & 4,229 & 3,909 & $\ldots$ \\
\hline Stay-over arrivals (thousands) & 515 & 545 & 507 & 498 & $\ldots$ \\
\hline Cruiseship arrivals (thousands) & 433 & 533 & 528 & 523 & $\ldots$ \\
\hline Average length of stay (days) $2 /$ & 7.6 & 7.3 & 7.3 & 6.8 & $\ldots$ \\
\hline Total bed capacity (thousands) & 19 & 18 & 19 & 22 & $\ldots$ \\
\hline Total bed occupancy rates (percent) & 57.7 & 60.4 & 53.0 & 42.7 & $\ldots$ \\
\hline Daily expenditure per head (in U.S. dollars) & 155.6 & 160.3 & 168.8 & 168.3 & $\ldots$ \\
\hline Total tourist expenditure (in millions of U.S. dollars) & 676 & 723 & 714 & 658 & $\ldots$ \\
\hline Stay-over tourists & 632 & 669 & 660 & 605 & $\ldots$ \\
\hline \multirow[t]{2}{*}{ Cruiseship tourists } & 44 & 54 & 54 & 53 & $\ldots$ \\
\hline & \multicolumn{3}{|c|}{ (Annual percentage change) } & & \\
\hline Total tourist days & 11.7 & 3.8 & -6.2 & -7.6 & $\ldots$ \\
\hline Stay-over visitors & 0.4 & 5.8 & -6.9 & -1.8 & $\ldots$ \\
\hline Cruiseship passengers & -14.6 & 23.2 & -1.1 & -0.8 & $\ldots$ \\
\hline Tourist expenditures & -3.8 & 6.9 & -1.2 & -7.8 & $\ldots$ \\
\hline \multirow[t]{2}{*}{ Daily expenditure per head } & -14.0 & 3.0 & 5.3 & -0.3 & $\cdots$ \\
\hline & \multicolumn{3}{|c|}{ (In percent of GDP } & & \\
\hline \multirow[t]{2}{*}{ Total tourist expenditure } & 27.2 & 27.9 & 27.8 & 25.3 & $\ldots$ \\
\hline & \multicolumn{3}{|c|}{ (In thousands) } & & \\
\hline Stay-over visitors by country of residence & 515 & 545 & 507 & 498 & $\ldots$ \\
\hline United States & 105 & 112 & 107 & 123 & $\ldots$ \\
\hline Canada & 57 & 60 & 52 & 47 & $\ldots$ \\
\hline CARICOM & 86 & 87 & 80 & 90 & $\ldots$ \\
\hline United Kingdom & 203 & 227 & 217 & 193 & $\ldots$ \\
\hline Other & 63 & 58 & 51 & 46 & $\cdots$ \\
\hline Stay-over visitors by intended length of stay & 515 & 545 & 507 & 498 & ... \\
\hline 1-3 days & 79 & 84 & 81 & 82 & $\ldots$ \\
\hline 4-7 days & 179 & 173 & 161 & 163 & $\ldots$ \\
\hline \multirow[t]{2}{*}{ Over 7 days } & 256 & 288 & 266 & 253 & $\cdots$ \\
\hline & \multicolumn{3}{|c|}{ (In percent of total arrivals) } & & \\
\hline \multicolumn{6}{|l|}{ Stay-over visitors by country of residence } \\
\hline United States & 20.4 & 20.6 & 21.0 & 24.8 & $\ldots$ \\
\hline Canada & 11.1 & 11.0 & 10.3 & 9.4 & $\ldots$ \\
\hline CARICOM & 16.7 & 16.1 & 15.8 & 18.0 & $\ldots$ \\
\hline United Kingdom & 39.4 & 41.6 & 42.9 & 38.7 & .. \\
\hline Other & 12.3 & 10.7 & 10.0 & 9.2 & $\ldots$ \\
\hline \multicolumn{6}{|l|}{ Stay-over visitors by intended length of stay } \\
\hline $1-3$ days & 15.4 & 15.4 & 16.0 & 16.4 & $\ldots$ \\
\hline $4-7$ days & 34.7 & 31.8 & 31.7 & 32.8 & $\ldots$ \\
\hline Over 7 days & 49.8 & 52.8 & 52.4 & 50.8 & $\ldots$ \\
\hline
\end{tabular}

Sources: Barbadian authorities; and Fund staff estimates.

1/ Stay-over arrivals multiplied by average length of stay, plus cruise-ship arrivals. Assumes average length of stay of tourists in establishments which are not surveyed is the same as that for surveyed establishments.

2/ Derived from a survey of establishments. Tourists staying in establishments that are not surveyed represent about one third of total stay-over arrivals. 
Table 5. Barbados: Retail Price Index

(May $1994=100)$

\begin{tabular}{|c|c|c|c|c|c|c|c|c|c|c|}
\hline & Total & Food & Tobacco & Housing & $\begin{array}{r}\text { Fuel } \\
\text { and } \\
\text { Light }\end{array}$ & $\begin{array}{r}\text { Household } \\
\text { Operations } \\
\text { and } \\
\text { Supplies }\end{array}$ & $\begin{array}{r}\text { Clothing } \\
\text { and } \\
\text { Footwear }\end{array}$ & $\begin{array}{l}\text { Trans- } \\
\text { portation }\end{array}$ & $\begin{array}{r}\text { Medical } \\
\text { and } \\
\text { Personal } \\
\text { Care }\end{array}$ & $\begin{array}{r}\text { Education } \\
\text { Recreation } \\
\text { and Mis- } \\
\text { cellaneous } \\
\text { Services }\end{array}$ \\
\hline \multirow[t]{2}{*}{ Weights } & 1.000 & 0.394 & 0.064 & 0.168 & 0.052 & 0.081 & 0.050 & 0.105 & 0.038 & 0.048 \\
\hline & \multicolumn{10}{|c|}{ (Averages) } \\
\hline 1990 & 88.3 & 96.2 & 85.5 & 77.1 & 96.7 & 86.5 & 95.5 & 73.3 & 79.5 & 92.2 \\
\hline 1991 & 93.9 & 100.8 & 92.5 & 85.6 & 97.0 & 90.1 & 98.1 & 86.0 & 82.3 & 93.2 \\
\hline 1992 & 99.5 & 101.0 & 99.1 & 100.1 & 99.7 & 99.6 & 101.1 & 97.6 & 90.7 & 94.0 \\
\hline 1993 & 100.7 & 101.0 & 98.1 & 100.2 & 102.8 & 100.4 & 102.5 & 102.8 & 98.4 & 97.0 \\
\hline 1994 & 100.6 & 101.2 & 99.6 & 100.0 & 100.6 & 99.5 & 101.5 & 100.8 & 99.8 & 99.2 \\
\hline 1995 & 103.0 & 106.1 & 101.2 & 103.7 & 104.0 & 98.0 & 97.4 & 99.3 & 101.0 & 100.3 \\
\hline 1996 & 105.5 & 110.2 & 104.2 & 105.7 & 103.7 & 98.7 & 96.8 & 100.5 & 103.6 & 102.7 \\
\hline 1997 & 113.6 & 125.1 & 112.4 & 107.0 & 112.9 & 103.6 & 91.4 & 108.6 & 101.7 & 114.4 \\
\hline 1998 & 112.2 & 119.7 & 113.8 & 110.6 & 109.8 & 102.0 & 90.4 & 108.9 & 102.6 & 119.6 \\
\hline 1999 & 113.9 & 122.7 & 114.8 & 113.4 & 106.8 & 101.0 & 93.6 & 109.9 & 101.9 & 122.3 \\
\hline 2000 & 116.7 & 125.8 & 116.0 & 116.5 & 122.5 & 95.1 & 91.1 & 106.6 & 112.0 & 126.6 \\
\hline \multirow[t]{2}{*}{2001} & 119.7 & 132.4 & 120.4 & 120.0 & 121.1 & 90.4 & 82.5 & 108.2 & 114.9 & 129.5 \\
\hline & \multicolumn{10}{|c|}{ (End of period) } \\
\hline \multicolumn{11}{|l|}{2000} \\
\hline January & 115.6 & 125.6 & 115.2 & 112.9 & 114.4 & 96.3 & 91.8 & 106.4 & 109.6 & 127.3 \\
\hline February & 114.8 & 122.6 & 113.8 & 115.0 & 115.0 & 97.8 & 92.9 & 105.6 & 109.8 & 127.7 \\
\hline March & 114.0 & 120.2 & 114.7 & 115.0 & 118.2 & 93.4 & 92.5 & 107.6 & 112.0 & 127.5 \\
\hline April & 113.7 & 120.0 & 114.7 & 115.0 & 119.4 & 93.3 & 92.3 & 105.9 & 110.7 & 125.5 \\
\hline May & 114.6 & 120.9 & 116.8 & 116.0 & 120.3 & 94.4 & 92.6 & 106.2 & 112.1 & 125.1 \\
\hline June & 114.9 & 121.6 & 116.1 & 116.0 & 120.9 & 96.1 & 90.8 & 106.8 & 112.3 & 125.4 \\
\hline July & 115.8 & 124.1 & 114.0 & 116.0 & 121.8 & 95.8 & 91.0 & 106.5 & 112.7 & 125.2 \\
\hline August & 117.3 & 127.1 & 114.3 & 118.2 & 122.8 & 94.5 & 90.1 & 107.0 & 112.9 & 125.5 \\
\hline September & 118.5 & 130.2 & 116.1 & 118.3 & 124.0 & 94.3 & 91.9 & 104.1 & 112.4 & 125.6 \\
\hline October & 119.9 & 132.6 & 117.9 & 118.2 & 125.8 & 95.0 & 90.0 & 107.7 & 113.1 & 124.3 \\
\hline November & 120.8 & 132.8 & 119.3 & 118.8 & 133.4 & 94.6 & 88.2 & 107.7 & 113.5 & 130.6 \\
\hline December & 120.4 & 131.7 & 118.8 & 118.8 & 133.4 & 95.4 & 88.6 & 107.4 & 113.4 & 130.0 \\
\hline \multicolumn{11}{|l|}{2001} \\
\hline January & 119.1 & 128.8 & 118.0 & 118.9 & 125.9 & 94.3 & 88.1 & 110.7 & 113.7 & 130.3 \\
\hline February & 117.9 & 126.9 & 117.2 & 120.0 & 124.8 & 93.1 & 85.1 & 108.1 & 114.9 & 130.4 \\
\hline March & 117.8 & 127.5 & 119.6 & 119.9 & 124.4 & 93.0 & 79.4 & 107.9 & 115.3 & 127.4 \\
\hline April & 118.4 & 128.4 & 121.5 & 119.9 & 124.0 & 90.5 & 82.9 & 109.4 & 115.2 & 127.7 \\
\hline May & 120.5 & 132.7 & 122.2 & 120.7 & 122.0 & 90.7 & 83.6 & 111.2 & 115.2 & 128.7 \\
\hline June & 120.7 & 133.5 & 121.9 & 120.7 & 122.8 & 90.6 & 81.7 & 110.8 & 115.1 & 128.6 \\
\hline July & 121.6 & 135.6 & 121.2 & 120.8 & 122.8 & 90.4 & 84.6 & 110.6 & 115.0 & 128.4 \\
\hline August & 120.8 & 136.5 & 120.0 & 119.5 & 118.7 & 89.5 & 84.4 & 105.0 & 114.1 & 130.3 \\
\hline September & 120.5 & 135.5 & 121.3 & 119.4 & 118.4 & 89.3 & 82.4 & 106.8 & 114.5 & 130.3 \\
\hline October & 120.2 & 135.2 & 122.3 & 119.3 & 117.6 & 88.1 & 81.4 & 106.2 & 115.7 & 130.3 \\
\hline November & 120.1 & 134.7 & 121.8 & 120.1 & 116.6 & 88.0 & 79.4 & 106.2 & 115.5 & 131.2 \\
\hline December & 118.9 & 133.2 & 117.9 & 120.2 & 115.3 & 86.7 & 77.5 & 105.8 & 115.1 & 130.9 \\
\hline \multirow[t]{2}{*}{ Weights 1/ } & 1.000 & 0.338 & 0.037 & 0.123 & 0.063 & 0.101 & 0.034 & 0.059 & 0.170 & 0.074 \\
\hline & \multicolumn{10}{|c|}{ (July $2001=100)$} \\
\hline 2002 & 99.6 & 100.9 & 97.6 & 101.2 & 100.9 & 94.1 & 92.6 & 98.4 & 105.9 & 99.9 \\
\hline 2003 (Sept.) & 100.8 & 102.7 & 98.4 & 100.0 & 99.7 & 92.4 & 91.3 & 101.5 & 109.5 & 103.1 \\
\hline
\end{tabular}

Source: Barbados Statistical Service.

1/ The Retail Price Index was revised from July 2001 with a new base period of July 2001=100, instead of May 1994=100. 
Table 6. Barbados: Selected Labor Statistics

(Household Survey Basis) 1/

\begin{tabular}{|c|c|c|c|c|c|}
\hline & 1999 & 2000 & 2001 & 2002 & 2003 \\
\hline & \multicolumn{5}{|c|}{ (In thouands) } \\
\hline Total population & 267.4 & 268.8 & 269.8 & 270.6 & $\ldots$ \\
\hline Total adult population $2 /$ & 205.7 & 207.4 & 208.8 & 209.4 & $\ldots$ \\
\hline Male & 95.1 & 95.1 & 98.7 & 99.1 & $\ldots$ \\
\hline Female & 106.7 & 112.3 & 110.1 & 110.3 & ... \\
\hline Labor force & 139.5 & 142.2 & 145.1 & 143.2 & $\ldots$ \\
\hline Male & 72.8 & 73.4 & 74.9 & 74.2 & $\ldots$ \\
\hline Female & 66.7 & 68.8 & 70.2 & 69.3 & $\ldots$ \\
\hline Employed & 125.1 & 129.0 & 130.8 & 128.7 & $\ldots$ \\
\hline Male & 67.2 & 68 & 68.9 & 67.7 & $\ldots$ \\
\hline Female & 57.9 & 61.0 & 61.9 & 60.9 & $\ldots$ \\
\hline Unemployed 3/ & 14.4 & 13.2 & 14.3 & 14.5 & $\ldots$ \\
\hline Male & 5.6 & 5.4 & 6.0 & 6.5 & $\ldots$ \\
\hline \multirow[t]{2}{*}{ Female } & 8.8 & 7.8 & 8.3 & 8.4 & $\ldots$ \\
\hline & \multicolumn{5}{|c|}{ (In percent) } \\
\hline Participation rate 4/ & 67.8 & 68.6 & 69.5 & 68.5 & $\ldots$ \\
\hline Male & 74.8 & 74.9 & 75.9 & 74.9 & $\ldots$ \\
\hline Female & 61.5 & 62.9 & 63.8 & 62.8 & $\ldots$ \\
\hline Employment by industry & 100.0 & 100.0 & 100.0 & 100.0 & $\ldots$ \\
\hline Agriculture and fishing & 4.3 & 3.8 & 4.2 & 4.0 & $\ldots$ \\
\hline Manufacturing & 8.3 & 8.1 & 7.1 & 7.0 & $\ldots$ \\
\hline Construction 5/ & 11.6 & 10.9 & 11.0 & 10.3 & $\ldots$ \\
\hline Electricity, gas, and water & 1.6 & 1.5 & 1.5 & 1.5 & $\ldots$ \\
\hline Commerce and tourism & 24.1 & 26.0 & 25.4 & 26.3 & $\ldots$ \\
\hline Transport, storage, and communications & 3.7 & 3.9 & 4.4 & 3.7 & $\ldots$ \\
\hline Financial institutions & 5.7 & 7.3 & 8.0 & 8.5 & $\ldots$ \\
\hline Government and other services & 40.7 & 38.6 & 38.3 & 38.9 & $\ldots$ \\
\hline Unemployment rate $6 /$ & 10.4 & 9.4 & 9.9 & 10.3 & $\ldots$ \\
\hline Male & 7.7 & 7.4 & 8.0 & 8.7 & $\ldots$ \\
\hline Female & 13.2 & 11.4 & 11.9 & 12.1 & $\ldots$ \\
\hline
\end{tabular}

Source: Barbados Statistical Service.

1/ Based on the 1990 population census and quarterly or semiannual labor force survey estimates. Results of the 1990 census are used to estimate the proportion of adult population in intercensus years.

2/ Persons 15 years of age or over, end of year.

3/ Unemployed persons comprise those actively seeking work with a three-month period prior to the date of interview.

4/ Employed and actively seeking work unemployed in relation to the labor force.

$5 /$ Includes mining and quarrying.

6/ Number of persons unemployed in relation to sum of number of workers employed and unemployed. 
Table 7. Barbados: Public Service Wage Increases 1/

(In percent)

\begin{tabular}{|c|c|c|c|}
\hline & Wage Increase & $\begin{array}{l}\text { Rate of Change } \\
\text { of Retail Price Index }\end{array}$ & Real Wage Increase \\
\hline 1984 & 15.0 & 4.6 & 9.9 \\
\hline 1985 & 5.0 & 1.3 & 3.6 \\
\hline 1986 & $1.4-9.0$ & 1.4 & $0.0-7.5$ \\
\hline 1987 & 2.0 & 2.7 & -0.7 \\
\hline 1988 & $5.0-8.2$ & 4.6 & $0.4-4.7$ \\
\hline 1989 & 2.0 & 5.9 & -3.7 \\
\hline 1990 & $3.5-7.0$ & 3.1 & $0.4-3.8$ \\
\hline 1991 & 3.0 & 6.4 & -10.4 \\
\hline October 1, $19912 /$ & -8.0 & & \\
\hline 1992 & 0.0 & 6.1 & -6.1 \\
\hline 1993 & 8.7 & 1.1 & 7.6 \\
\hline 1994 & 0.0 & 0.7 & -0.7 \\
\hline 1995 & 3.0 & 1.9 & 1.1 \\
\hline 1996 & 3.0 & 2.4 & 0.6 \\
\hline 1997 & 6.3 & 7.7 & -1.4 \\
\hline 1998 & 3.0 & -1.3 & 4.3 \\
\hline 1999 & 4.2 & 1.6 & 2.6 \\
\hline 2000 & 3.0 & 2.4 & 0.6 \\
\hline 2001 & 3.0 & 2.8 & 0.2 \\
\hline 2002 & 3.0 & 0.2 & 2.8 \\
\hline 2003 & 2.0 & 1.5 & 0.5 \\
\hline
\end{tabular}

Sources: World Bank, Barbados-Requirements for Industrial Development, 1991; Ministry of Finance and Economic Affairs; and Barbados Statistical Service.

1/ Beginning in 1994, the reported wage increases are estimated.

2/ A nominal wage cut as part of the fiscal adjustment in the context of a 16-month stand-by arrangement supported by the IMF. 
Table 8. Barbados: Central Government Operations 1/

\begin{tabular}{|c|c|c|c|c|c|}
\hline & $1998 / 99$ & $1999 / 00$ & $2000 / 01$ & $2001 / 02$ & $2002 / 03$ \\
\hline & \multicolumn{5}{|c|}{ (In millions of Barbados dollars) } \\
\hline Budgetary revenue & 1,530 & 1,610 & 1,705 & 1,703 & 1,716 \\
\hline Tax revenue & 1,464 & 1,515 & 1,615 & 1,613 & 1,620 \\
\hline Nontax revenue & 66 & 94 & 90 & 90 & 96 \\
\hline Budgetary expenditure & 1,576 & 1,693 & 1,803 & 1,917 & 1,997 \\
\hline Current expenditure & 1,329 & 1,427 & 1,502 & 1,571 & 1,672 \\
\hline Wages and salaries and NIS contributions & 551 & 603 & 626 & 630 & 666 \\
\hline \multicolumn{6}{|l|}{ Of which } \\
\hline National insurance contribution & 35 & 37 & 38 & 39 & 41 \\
\hline Goods and services & 154 & 163 & 187 & 203 & 211 \\
\hline Interest & 219 & 251 & 219 & 253 & 274 \\
\hline Domestic & 170 & 190 & 142 & 170 & 174 \\
\hline Of which: external & 49 & 61 & 77 & 83 & 100 \\
\hline Transfers & 406 & 411 & 471 & 486 & 521 \\
\hline Capital expenditure and net lending & 246 & 266 & 300 & 346 & 325 \\
\hline Of which: net lending & -8 & 3 & 11 & 12 & 6 \\
\hline Budgetary balance & -46 & -83 & -98 & -214 & -281 \\
\hline Float and extra-budgetary expenditure 2 / & -10 & -19 & 23 & 36 & 217 \\
\hline Overall balance & -35 & -64 & -120 & -250 & -498 \\
\hline Financing & 46 & 64 & 120 & 250 & 498 \\
\hline Foreign financing (net) & -42 & 96 & 215 & 322 & -19 \\
\hline Disbursements & 40 & 201 & 255 & 364 & 58 \\
\hline Amortization & -82 & -105 & -40 & -42 & -78 \\
\hline Domestic financing & 87 & -32 & -147 & -72 & 517 \\
\hline Banking system & 18 & -110 & -109 & -108 & 413 \\
\hline Rest of financial system & 0 & 0 & 8 & -16 & 0 \\
\hline National Insurance Scheme & 59 & 79 & -45 & 52 & 105 \\
\hline \multirow[t]{2}{*}{ Privatization proceeds } & 0 & 0 & 52 & 0 & 0 \\
\hline & \multicolumn{5}{|c|}{ (In percent of GDP) } \\
\hline Budgetary revenue & 31.8 & 32.1 & 33.0 & 33.0 & 32.7 \\
\hline Tax revenue & 30.5 & 30.2 & 31.2 & 31.3 & 30.9 \\
\hline Nontax revenue & 1.4 & 1.9 & 1.7 & 1.8 & 1.8 \\
\hline Budgetary expenditure & 32.8 & 33.7 & 34.9 & 37.2 & 38.1 \\
\hline Current expenditure & 27.7 & 28.4 & 29.0 & 30.5 & 31.9 \\
\hline Capital expenditure and net lending & 5.1 & 5.3 & 5.8 & 6.7 & 6.2 \\
\hline Budgetary balance & -1.0 & -1.7 & -1.9 & -4.1 & -5.4 \\
\hline Float and extra-budgetary expenditure $2 /$ & -0.2 & -0.4 & 0.4 & 0.7 & 4.1 \\
\hline Overall balance & -0.7 & -1.3 & -2.3 & -4.9 & -9.5 \\
\hline Of which : primary balance & 3.6 & 3.7 & 1.9 & 0.1 & -4.3 \\
\hline Financing & 0.7 & 1.3 & 2.3 & 4.9 & 9.5 \\
\hline \multicolumn{6}{|l|}{ Memorandum items: } \\
\hline Overall balance of rest of nonfinancial public sector 3 / & -1.6 & 0.0 & -0.8 & -0.4 & -0.9 \\
\hline Nonfinancial public sector overall balance & -2.4 & -1.2 & -3.1 & -5.2 & -10.4 \\
\hline Government and government guaranteed debt & 63.6 & 68.1 & 73.3 & 81.6 & 83.8 \\
\hline Of which: central government debt & 57.9 & 58.5 & 63.4 & 72.4 & 75.7 \\
\hline Fiscal year GDP at market prices & 4,807 & 5,018 & 5,172 & 5,154 & 5,246 \\
\hline
\end{tabular}

Sources: Ministry of Finance; and Fund staff estimates.

1/ Fiscal years (April-March). Ratios expressed relative to fiscal-year GDP.

2 / Represents the difference between the budgetary deficit measured from above the line and the cash deficit measured on the basis of identified financing flows. Includes statistical discrepancy.

3/ After government transfers, based on financing data. Excludes the NIS. 
Table 9. Barbados: Central Government Revenue and Grants 1/

(In millions of Barbados dollars)

\begin{tabular}{|c|c|c|c|c|c|}
\hline & 1998/99 & $1999 / 00$ & $2000 / 01$ & $2001 / 02$ & $2002 / 03$ \\
\hline Total revenue and grants & $1,530.1$ & $1,609.8$ & $1,705.0$ & 1,703.1 & $1,716.0$ \\
\hline Current revenue & $1,530.1$ & $1,609.8$ & $1,705.0$ & $1,703.1$ & $1,716.0$ \\
\hline Tax revenue & $1,464.2$ & $1,515.4$ & $1,615.4$ & $1,612.9$ & $1,619.9$ \\
\hline Taxes on net income and profits & 459.9 & 512.9 & 591.6 & 571.4 & 604.7 \\
\hline Companies & 171.9 & 223.1 & 248.0 & 222.7 & 220.3 \\
\hline Individuals & 268.0 & 272.2 & 313.3 & 316.7 & 358.3 \\
\hline Other & 20.0 & 17.6 & 30.3 & 32.0 & 26.1 \\
\hline Levies & 27.1 & 28.6 & 28.0 & 28.6 & 29.0 \\
\hline Employment levy 2/ & 0.7 & 0.0 & 0.0 & 0.0 & 0.0 \\
\hline Training levy & 16.7 & 17.5 & 17.0 & 16.5 & 17.8 \\
\hline Environment levy & 9.7 & 11.1 & 11.1 & 10.2 & 11.2 \\
\hline Taxes on property & 77.7 & 98.3 & 93.7 & 102.5 & 95.7 \\
\hline Land tax & 55.1 & 62.2 & 64.6 & 63.1 & 66.2 \\
\hline Property transfer tax and other & 22.6 & 36.1 & 29.1 & 39.4 & 29.5 \\
\hline Taxes on domestic goods and services & 747.9 & 727.8 & 751.2 & 749.5 & 718.4 \\
\hline Value-added tax & 454.9 & 457.4 & 493.9 & 498.6 & 494.6 \\
\hline Consumption tax & 0.2 & 0.5 & 0.0 & 0.0 & 0.0 \\
\hline Highway revenue & 24.5 & 25.1 & 26.2 & 27.3 & 28.5 \\
\hline Hotel and restaurant sales tax & 0.5 & 0.7 & 0.7 & 0.6 & 1.0 \\
\hline Airport services & 15.1 & 15.5 & 17.9 & 16.4 & 15.8 \\
\hline Other & 252.7 & 228.7 & 213.7 & 206.6 & 178.5 \\
\hline Taxes on international trade & 151.6 & 147.9 & 149.5 & 160.9 & 172.1 \\
\hline Import duties & 136.9 & 134.6 & 135.4 & 146.4 & 160.1 \\
\hline Stamp duties & 14.7 & 13.3 & 14.1 & 14.5 & 12.0 \\
\hline Nontax revenue & 65.9 & 94.4 & 89.6 & 90.2 & 96.1 \\
\hline Property income & 4.8 & 6.4 & 15.3 & 15.5 & 21.9 \\
\hline \multicolumn{6}{|l|}{ Of which } \\
\hline Central bank profits & 0.0 & 0.0 & 9.5 & 12.7 & 14.5 \\
\hline Other nontax revenue & 61.1 & 88.0 & 74.6 & 73.5 & 74.2 \\
\hline Capital revenue and grants & 0.0 & 0.0 & 0.0 & 0.0 & 0.0 \\
\hline
\end{tabular}

Sources: Ministry of Finance; and Fund staff estimates.

1/ Fiscal years (April-March).

2/ Includes Public Employees' Fund. 
Table 10. Barbados: Central Government Transfers 1/

(In millions of Barbados dollars)

\begin{tabular}{|c|c|c|c|c|c|}
\hline & 1998/99 & 1999/00 & $2000 / 01$ & $2001 / 02$ & $2002 / 03$ \\
\hline Current transfers & 405.6 & 410.5 & 470.8 & 485.7 & 521.0 \\
\hline Nonfinancial public enterprises & 53.4 & 45.9 & 60.3 & 65.5 & 61.8 \\
\hline National Housing Corporation & 0.0 & 0.0 & 0.0 & 4.1 & 0.0 \\
\hline Post Office & 5.3 & 2.6 & 4.0 & 10.7 & 15.6 \\
\hline Civil Aviation (net) & 4.5 & 3.6 & 6.7 & 8.3 & 0.0 \\
\hline Barbados Investment and Development Corporation & 12.4 & 9.8 & 10.0 & 13.5 & 11.7 \\
\hline Transport Board & 10.3 & 7.6 & 17.2 & 6.4 & 9.9 \\
\hline Caribbean Broadcasting Corporation & 0.0 & 0.0 & 0.0 & 0.0 & 0.0 \\
\hline National Conservation Commission & 20.9 & 22.3 & 22.4 & 22.5 & 24.5 \\
\hline Printing Office & 2.5 & 2.8 & 1.9 & 2.6 & 3.7 \\
\hline Public financial institutions & 0.0 & 0.0 & 0.0 & 0.0 & $\mathbf{0 . 0}$ \\
\hline Pensions (contributory) & 88.3 & 95.3 & 101.0 & 111.6 & 118.7 \\
\hline Other public institutions & 211.6 & 203.9 & 246.8 & 243.9 & 256.9 \\
\hline Barbados defense and police & 27.9 & 28.7 & 27.6 & 28.6 & 32.6 \\
\hline Schools, colleges, and the University of the West Indies & 84.2 & 72.2 & 108.7 & 106.3 & 96.3 \\
\hline Tourism promotion & 44.5 & 43.3 & 49.1 & 51.0 & 60.4 \\
\hline Sanitation and public health service & 20.3 & 22.0 & 23.4 & 22.8 & 25.6 \\
\hline Export promotion & 0.0 & 0.0 & 0.0 & 0.0 & 0.0 \\
\hline Welfare and other agencies & 27.9 & 29.1 & 29.1 & 26.6 & 32.4 \\
\hline National Sports Council & 6.8 & 8.6 & 8.9 & 8.6 & 9.7 \\
\hline Nonprofit institutions (child care) & 11.6 & 12.7 & 13.7 & 13.0 & 14.3 \\
\hline Other nonprofit institutions & 3.0 & 9.9 & 3.5 & 3.7 & 20.6 \\
\hline Other transfers to individuals & 26.1 & 27.4 & 33.1 & 33.7 & 34.5 \\
\hline Welfare grants (including NAB) & 5.1 & 5.5 & 6.8 & 7.8 & 7.6 \\
\hline Scholarship bursaries and examinations & 15.0 & 11.2 & 14.6 & 17.4 & 21.2 \\
\hline Schools bus fares & 2.1 & 2.1 & 2.0 & 2.0 & 5.7 \\
\hline Other & 3.9 & 8.6 & 9.7 & 6.5 & 0.0 \\
\hline Sugar industry & 0.0 & 0.0 & 0.0 & 0.0 & 0.0 \\
\hline Subscriptions and contributions & 9.1 & 12.6 & 10.5 & 11.7 & 10.5 \\
\hline $\begin{array}{l}\text { Other, including contributions to regional international } \\
\text { organizations }\end{array}$ & 0.0 & 0.0 & 0.0 & 0.0 & $\mathbf{0 . 0}$ \\
\hline Capital transfers & 67.8 & 73.0 & 76.1 & 73.7 & 80.0 \\
\hline Nonfinancial public enterprises & 23.2 & 27.8 & 52.6 & 69.6 & 76.9 \\
\hline National Housing Corporation & 4.9 & 2.5 & 2.1 & 4.1 & 3.6 \\
\hline Barbados Agricultural Development and Marketing Corporation & 5.0 & 5.0 & 0.0 & 0.0 & 0.0 \\
\hline Barbados Agricultural Management Co. Ltd. & 10.0 & 19.3 & 19.3 & 19.3 & 0.0 \\
\hline Civil Aviation & 0.0 & 0.0 & 0.0 & 0.0 & 21.1 \\
\hline Barbados Water Authority & 0.0 & 0.0 & 0.0 & 0.0 & 0.0 \\
\hline Barbados Investment and Development Corporation & 2.5 & 1.0 & 2.5 & 0.1 & 0.9 \\
\hline Transport Board & 0.8 & 0.0 & 0.0 & 0.0 & 0.0 \\
\hline Other & 0.0 & 0.0 & 28.7 & 46.1 & 51.4 \\
\hline Public financial institutions & 0.9 & 7.0 & 2.2 & 2.4 & $\mathbf{0 . 0}$ \\
\hline Other public institutions & 32.2 & 36.9 & 19.3 & 0.0 & 0.0 \\
\hline Tourism and sports & 1.6 & 1.3 & 0.6 & 1.7 & 3.1 \\
\hline Abroad & 9.9 & 0.0 & 1.4 & 0.0 & 0.0 \\
\hline
\end{tabular}

Sources: Ministry of Finance; and Fund staff estimates.

1/ Fiscal years (April-March). 
Table 11 Barbados: Operations of the National Insurance Scheme 1/

(In millions of Barbados dollars)

\begin{tabular}{|c|c|c|c|c|c|}
\hline & 1998/99 & $1999 / 00$ & $2000 / 01$ & $2001 / 02$ & $2002 / 03$ \\
\hline Current revenue & 359.3 & 386.7 & 382.6 & 402.9 & 411.5 \\
\hline Contributions & 272.4 & 284.0 & 290.7 & 307.0 & 297.6 \\
\hline \multicolumn{6}{|l|}{ Of which } \\
\hline Central government insurance contributions & 34.7 & 37.3 & 38.3 & 38.7 & 40.5 \\
\hline Interest, dividends, etc. & 86.9 & 102.7 & 91.9 & 95.9 & 113.9 \\
\hline Current expenditure & 272.6 & 287.0 & 263.4 & 283.2 & 288.1 \\
\hline Benefits & 255.4 & 267.9 & 243.1 & 263.8 & 261.4 \\
\hline Administration & 17.2 & 19.1 & 20.4 & 19.5 & 26.7 \\
\hline Current surplus & 86.7 & 99.7 & 119.2 & 119.7 & 123.4 \\
\hline Capital expenditure & 0.0 & -0.2 & 0.0 & 0.0 & $\mathbf{0 . 0}$ \\
\hline Overall surplus & 86.7 & 99.9 & 119.2 & 119.7 & 123.4 \\
\hline Total financing & -86.7 & -99.9 & -119.2 & -119.7 & -123.4 \\
\hline External financing & -22.0 & -21.0 & -6.7 & -9.4 & 3.2 \\
\hline Domestic financing & -64.7 & -78.9 & -112.5 & -110.3 & -126.6 \\
\hline Financial intermediaries & 6.9 & -30.3 & -54.1 & -39.0 & 5.8 \\
\hline Treasury bills & 11.6 & -39.2 & 77.4 & 0.0 & 0.0 \\
\hline Central government debentures & -41.0 & -18.3 & -60.8 & -38.3 & -104.6 \\
\hline Other & -42.2 & 8.9 & -74.9 & -33.0 & -27.8 \\
\hline
\end{tabular}

Sources: National Insurance Scheme; and Central Bank of Barbados.

1/ Fiscal years (April-March). 
Table 12. Barbados: National Insurance Scheme: Investments and Cash Balances (In millions of Barbados dollars)

\begin{tabular}{|c|c|c|c|c|c|}
\hline & 1999 & 2000 & 2001 & 2002 & 2003 \\
\hline Total assets & $1,124.1$ & $1,246.3$ & $1,383.7$ & $1,486.4$ & $\ldots$ \\
\hline Cash balances & 5.5 & 12.5 & 33.8 & 9.7 & $\ldots$ \\
\hline Deposits & 169.8 & 208.5 & 235.8 & 239.9 & ... \\
\hline Domestic investments & 875.9 & 933.3 & $1,016.2$ & $1,140.7$ & $\ldots$ \\
\hline Debentures & 656.3 & 701.8 & 742.4 & 855.7 & ... \\
\hline Treasury bills & 45.3 & 0.0 & 0.0 & 0.0 & $\ldots$ \\
\hline Other & 174.3 & 231.4 & 273.9 & 285.0 & $\ldots$ \\
\hline Foreign assets & 72.9 & 92.0 & 97.8 & 96.1 & $\ldots$ \\
\hline
\end{tabular}

Source: Central Bank of Barbados. 
Table 13. Barbados: Public Sector Financing Requirement and Debt

\begin{tabular}{|c|c|c|c|c|c|}
\hline & 1998/99 & $1999 / 00$ & $2000 / 01$ & $2001 / 02$ & $2002 / 03$ \\
\hline & \multicolumn{5}{|c|}{ (In millions of Barbados dollars) } \\
\hline \multicolumn{6}{|l|}{ Gross financing requirement } \\
\hline Consolidated public sector deficit & 114 & 62 & 162 & 269 & 544 \\
\hline Central government & 35 & 64 & 120 & 250 & 498 \\
\hline Rest of the public sector $1 /$ & 78 & -2 & 42 & 19 & 46 \\
\hline Amortization payments on the public debt & 966 & 969 & 903 & 926 & 1,014 \\
\hline Domestic & 884 & 864 & 863 & 884 & 937 \\
\hline External & 82 & 105 & 40 & 42 & 78 \\
\hline Gross financing requirement & 1,079 & 1,031 & 1,065 & 1,195 & 1,558 \\
\hline Disbursements of domestic debt & 1,026 & 873 & 1,029 & 1,004 & 1,195 \\
\hline Drawdown of deposits & 13 & -43 & -271 & -173 & 304 \\
\hline Disbursements of external debt & 40 & 201 & 255 & 364 & 58 \\
\hline Privatization proceeds & 0 & 0 & 52 & 0 & 0 \\
\hline \multicolumn{6}{|l|}{ End-of-period public debt } \\
\hline Government and government-guaranteed debt & 3,055 & 3,415 & 3,789 & 4,204 & 4,398 \\
\hline Central government & 2,788 & 2,934 & 3,278 & 3,733 & 3,969 \\
\hline Rest of the public sector $1 /$ & 267 & 481 & 511 & 471 & 429 \\
\hline Domestic & 2,286 & 2,489 & 2,625 & 2,719 & 2,984 \\
\hline External & 770 & 927 & 1,165 & 1,485 & 1,414 \\
\hline & \multicolumn{5}{|c|}{ (In percent of GDP) } \\
\hline \multicolumn{6}{|l|}{ Gross financing requirement } \\
\hline Consolidated public sector deficit & 2.4 & 1.2 & 3.1 & 5.2 & 10.4 \\
\hline Central government & 0.7 & 1.3 & 2.3 & 4.9 & 9.5 \\
\hline Rest of the public sector $1 /$ & 1.6 & 0.0 & 0.8 & 0.4 & 0.9 \\
\hline Amortization payments on the public debt & 20.1 & 19.3 & 17.5 & 18.0 & 19.3 \\
\hline Domestic & 18.4 & 17.2 & 16.7 & 17.2 & 17.9 \\
\hline External & 1.7 & 2.1 & 0.8 & 0.8 & 1.5 \\
\hline Gross financing requirement & 22.5 & 20.5 & 20.6 & 23.2 & 29.7 \\
\hline Disbursements of domestic debt & 21.4 & 17.4 & 19.9 & 19.5 & 22.8 \\
\hline Drawdown of deposits & 0.3 & -0.8 & -5.2 & -3.4 & 5.8 \\
\hline Disbursements of external debt & 0.8 & 4.0 & 4.9 & 7.1 & 1.1 \\
\hline Privatization proceeds & 0.0 & 0.0 & 1.0 & 0.0 & 0.0 \\
\hline \multicolumn{6}{|l|}{ End-of-period public debt } \\
\hline Government and government-guaranteed debt & 63.6 & 68.1 & 73.3 & 81.6 & 83.8 \\
\hline Central government & 58.0 & 58.5 & 63.4 & 72.4 & 75.7 \\
\hline Rest of the public sector $1 /$ & 5.6 & 9.6 & 9.9 & 9.1 & 8.2 \\
\hline Domestic & 47.6 & 49.6 & 50.8 & 52.8 & 56.9 \\
\hline External & 16.0 & 18.5 & 22.5 & 28.8 & 27.0 \\
\hline
\end{tabular}

Sources: Barbadian authorities; and Fund staff estimates and projections.

1/ Excludes the National Insurance Scheme. 
Table 14. Barbados: Operations of Selected Nonfinancial Public Enterprises 1/

(In millions of Barbados dollars)

\begin{tabular}{|c|c|c|c|c|c|}
\hline & 1998/99 & 1999/00 & $2000 / 01$ & $2001 / 02$ & $\begin{array}{r}\text { Prel. } \\
2002 / 03\end{array}$ \\
\hline \multicolumn{6}{|c|}{ I. National Housing Corporation } \\
\hline Current revenue & 19.2 & 22.6 & 29.5 & 29.5 & 32.3 \\
\hline Transfers from central government & 0.0 & 0.0 & 0.0 & 4.1 & 3.6 \\
\hline Current expenditure & 16.5 & 27.6 & 25.9 & 25.9 & 26.3 \\
\hline Current surplus or deficit (-) & 2.7 & -5.0 & 3.6 & 3.6 & 6.0 \\
\hline Capital revenue 2/ & 4.9 & 5.6 & 5.6 & 5.6 & 0.0 \\
\hline Transfers from central government $2 /$ & 4.9 & 2.5 & 2.1 & 4.1 & 5.1 \\
\hline Capital expenditure 2/ & 9.7 & 2.7 & 2.7 & 2.7 & 10.4 \\
\hline Overall surplus or deficit (-) & -2.1 & -2.1 & 6.5 & 6.5 & 0.7 \\
\hline \multicolumn{6}{|c|}{ II. National Petroleum Corporation } \\
\hline Current revenue & 15.5 & 15.3 & 12.5 & 11.2 & 13.3 \\
\hline Current expenditure & 10.8 & 12.2 & 10.3 & 9.5 & 11.7 \\
\hline Current surplus or deficit (-) & 4.7 & 3.1 & 2.2 & 1.7 & 1.6 \\
\hline Capital expenditure & 10.0 & 2.5 & 2.0 & 1.8 & 2.3 \\
\hline Overall surplus or deficit (-) & -5.3 & 0.6 & 0.2 & -0.1 & -0.7 \\
\hline \multicolumn{6}{|c|}{ III. Barbados National Oil Company } \\
\hline Current revenue & 44.1 & 208.3 & 281.5 & 256.5 & 82.8 \\
\hline Current expenditure & 42.7 & 205.3 & 259.5 & 223.5 & 83.3 \\
\hline Other & 0.0 & 0.0 & 0.0 & 0.0 & 0.0 \\
\hline Current surplus or deficit (-) & 1.4 & 3.0 & 22.0 & 33.0 & -0.5 \\
\hline Capital expenditure & 0.0 & 0.0 & 0.2 & 1.8 & 0.4 \\
\hline Overall surplus or deficit (-) & 1.4 & 3.0 & 21.8 & 31.2 & -0.9 \\
\hline \multicolumn{6}{|c|}{ IV. Civil Aviation } \\
\hline Current revenue & 11.9 & 13.0 & 26.1 & 28.9 & 0.3 \\
\hline Transfers from central government & 4.5 & 3.6 & 6.7 & 8.3 & 0.0 \\
\hline Current expenditure & 16.4 & 16.6 & 19.4 & 22.9 & 0.8 \\
\hline Current surplus or deficit (-) & -4.5 & -3.6 & 6.7 & 6.0 & -0.5 \\
\hline $\begin{array}{l}\text { Capital revenue } \\
\text { Transfers from central government }\end{array}$ & 0.0 & 0.0 & 0.0 & 10.7 & 25.0 \\
\hline Capital expenditure & 0.0 & 0.0 & 3.7 & 16.7 & 14.4 \\
\hline Overall surplus or deficit (-) & -4.5 & -3.6 & 3.0 & -10.7 & 10.1 \\
\hline \multicolumn{6}{|c|}{ V. Barbados Water Authority } \\
\hline Current revenues & 53.2 & 62.1 & 54.3 & 55.2 & 62.7 \\
\hline Current expenditure & 51.8 & 61.9 & 56.1 & 67.3 & 71.4 \\
\hline Current surplus or deficit (-) & 1.4 & 0.2 & -1.8 & -12.1 & -8.7 \\
\hline
\end{tabular}


Table 14. Barbados: Operations of Selected Nonfinancial Public Enterprises (In millions of Barbados dollars)

\begin{tabular}{|c|c|c|c|c|c|}
\hline & 1998/99 & $1999 / 00$ & $2000 / 01$ & $2001 / 02$ & $\begin{array}{r}\text { Prel. } \\
2002 / 03\end{array}$ \\
\hline Capital revenue & 0.0 & 0.0 & 1.1 & 0.7 & 0.0 \\
\hline Transfers from central government & 0.0 & 0.0 & 1.1 & 0.7 & 0.0 \\
\hline Capital expenditure & 15.8 & 7.8 & 2.5 & 50.7 & 5.5 \\
\hline Overall surplus or deficit (-) & -14.4 & -7.6 & -4.3 & -62.2 & -14.2 \\
\hline \multicolumn{6}{|c|}{ VI. Post Office } \\
\hline Current revenue & 13.0 & 16.8 & 23.8 & 22.0 & 26.9 \\
\hline Transfers from central government & 5.3 & 2.6 & 4.0 & 10.7 & 15.6 \\
\hline Current expenditure & 18.3 & 19.4 & 21.3 & 21.7 & 25.3 \\
\hline Current surplus or deficit (-) & -5.3 & -2.6 & 2.5 & 0.3 & 1.6 \\
\hline Capital revenue & 0.0 & 0.0 & 0.0 & 7.1 & 0.0 \\
\hline Transfers from central government & 0.0 & 0.0 & 0.0 & 7.1 & \\
\hline Capital expenditure & 0.0 & 0.0 & 0.0 & 1.7 & 0.0 \\
\hline Overall surplus or defïcit (-) & -5.3 & -2.6 & 2.5 & -1.4 & 1.6 \\
\hline \multicolumn{6}{|c|}{ VII. Barbados Industrial Development Corporation } \\
\hline Current revenue & 22.4 & 19.0 & 20.7 & 22.6 & 26.2 \\
\hline Transfers from central government & 12.4 & 9.8 & 10.0 & 13.5 & 15.7 \\
\hline Current expenditure & 16.3 & 19.2 & 20.6 & 20.8 & 23.5 \\
\hline Current surplus or deficit (-) & 6.1 & -0.2 & 0.1 & 1.8 & 2.7 \\
\hline Capital revenue & 3.3 & 2.8 & 7.5 & 1.7 & 2.9 \\
\hline Transfers from central government & 2.5 & 1.0 & 2.5 & 0.1 & 0.9 \\
\hline Capital expenditure & 7.0 & 10.5 & 7.9 & 2.1 & 1.6 \\
\hline Overall surplus or deficit (-) & 2.4 & -7.9 & -0.3 & 1.4 & 4.0 \\
\hline \multicolumn{6}{|c|}{ VIII. Transport Board } \\
\hline Current revenue & 45.8 & 27.6 & 33.2 & 37.9 & 41.5 \\
\hline Transfers from central government & 10.3 & 7.6 & 17.2 & 6.4 & 4.9 \\
\hline Current expenditure & 54.6 & 29.0 & 52.9 & 58.2 & 59.6 \\
\hline Current surplus or deficit (-) & -8.8 & -1.4 & -19.7 & -20.3 & -18.1 \\
\hline Capital revenue & 0.8 & 0.0 & 0.0 & 0.0 & 23.1 \\
\hline Transfers from central government & 0.8 & 0.0 & 0.0 & 0.0 & 23.1 \\
\hline Capital expenditure & 0.1 & 10.0 & 14.8 & 0.4 & 1.6 \\
\hline Overall surplus or deficit (-) & -8.1 & -11.4 & -34.6 & -20.6 & 3.4 \\
\hline
\end{tabular}


Table 14. Barbados: Operations of Selected Nonfinancial Public Enterprises

(In millions of Barbados dollars)

\begin{tabular}{|c|c|c|c|c|c|}
\hline & 1998/99 & $1999 / 00$ & $2000 / 01$ & $2001 / 02$ & $\begin{array}{r}\text { Prel. } \\
2002 / 03\end{array}$ \\
\hline \multicolumn{6}{|c|}{ IX. Port Authority } \\
\hline Current revenue & 66.2 & 68.9 & 72.7 & 52.0 & 67.1 \\
\hline Current expenditure & 71.5 & 66.7 & 62.7 & 50.7 & 66.9 \\
\hline Current surplus or deficit (-) & -5.3 & 2.2 & 10.0 & 1.3 & 0.2 \\
\hline Capital revenue & 5.8 & 0.0 & 0.0 & 0.0 & 0.0 \\
\hline Capital expenditure & 0.9 & 2.6 & 2.7 & 1.7 & 8.5 \\
\hline Overall surplus or deficit (-) & -0.4 & -0.4 & 7.3 & -0.4 & -8.3 \\
\hline \multicolumn{6}{|c|}{ X. Barbados Agricultural Management Co. Ltd. } \\
\hline $\begin{array}{l}\text { Current revenue } \\
\text { Transfers from central government }\end{array}$ & 60.8 & 62.2 & 51.2 & 51.2 & 57.2 \\
\hline Current expenditure & 71.5 & 74.5 & 73.8 & 73.8 & 74.0 \\
\hline Current surplus or deficit (-) & -10.7 & -12.2 & -22.6 & -22.6 & -16.8 \\
\hline Capital revenue & 10.0 & 21.4 & 19.4 & 19.4 & 9.9 \\
\hline Transfers from central government & 10.0 & 19.3 & 19.3 & 19.3 & 9.9 \\
\hline Capital expenditure & & & 2.8 & 2.8 & 7.7 \\
\hline Overall surplus or deficit (-) & -0.7 & 9.1 & -6.0 & -6.0 & -14.6 \\
\hline \multicolumn{6}{|c|}{ XI. Hotels and Resorts, Ltd. (Gems of Barbados Project) } \\
\hline $\begin{array}{l}\text { Current revenue } \\
\text { Transfers from central government }\end{array}$ & $\cdots$ & 10.6 & 13.4 & 13.4 & 18.7 \\
\hline Current expenditure & $\cdots$ & 19.8 & 29.7 & 29.7 & 22.6 \\
\hline Current surplus or deficit (-) & $\cdots$ & -9.1 & -16.3 & -16.3 & -3.9 \\
\hline $\begin{array}{l}\text { Capital revenue } \\
\text { Transfers from central government }\end{array}$ & $\begin{array}{l}\cdots \\
\cdots\end{array}$ & 0.1 & 0.0 & 0.0 & 0.0 \\
\hline Capital expenditure & $\cdots$ & 7.7 & 25.4 & 25.4 & 7.4 \\
\hline Overall surplus or deficit (-) & $\cdots$ & -16.7 & -41.6 & -41.6 & -11.3 \\
\hline \multicolumn{6}{|c|}{ XII. Needhams Point Development--Hilton (Consortium) and Bridgetown Port Expansion } \\
\hline Current revenue & $\ldots$ & $\ldots$ & $\ldots$ & $\ldots$ & 3.1 \\
\hline Transfers from central government & $\cdots$ & $\cdots$ & $\cdots$ & $\cdots$ & \\
\hline Current expenditure & $\begin{array}{l}\cdots \\
\cdots\end{array}$ & $\begin{array}{l}\cdots \\
\cdots\end{array}$ & $\begin{array}{l}\cdots \\
\cdots\end{array}$ & $\begin{array}{l}\cdots \\
\cdots\end{array}$ & 0.9 \\
\hline Current surplus or deficit (-) & $\begin{array}{l}\ldots \\
\ldots\end{array}$ & $\begin{array}{l}\ldots \\
\ldots\end{array}$ & $\begin{array}{l}\ldots \\
\ldots\end{array}$ & $\begin{array}{l}\ldots \\
\ldots\end{array}$ & 2.3 \\
\hline & $\ldots$ & $\ldots$ & $\ldots$ & $\ldots$ & \\
\hline Capital revenue & $\cdots$ & $\cdots$ & $\cdots$ & $\cdots$ & 30.0 \\
\hline Transfers from central government & $\cdots$ & $\cdots$ & $\cdots$ & $\cdots$ & 30.0 \\
\hline Capital expenditure & $\begin{array}{l}\ldots \\
\ldots\end{array}$ & $\begin{array}{l}\cdots \\
\ldots\end{array}$ & $\begin{array}{l}\ldots \\
\ldots\end{array}$ & 3.6 & 38.4 \\
\hline Overall surplus or deficit (-) & $\ldots$ & $\ldots$ & $\ldots$ & $\ldots$ & -6.1 \\
\hline
\end{tabular}

Sources: Ministry of Finance: annual reports and financial statements of the enterprises; and Fund staff estimates.

1/ Fiscal years (April-March).

2/ Includes an estimate for the National Housing Corporation for FY 2000/01 and FY 2001/02.

3/ Includes an estimate for the Barbados Agricultural Management Corporation for FY 2001/02.

4/ Includes an estimate for Hotels and Resorts, Ltd. for FY 2001/02. 
Table 15. Barbados: Summary Accounts of the Consolidated Financial System

\begin{tabular}{|c|c|c|c|c|c|}
\hline & 1999 & 2000 & 2001 & 2002 & 2003 \\
\hline & \multicolumn{5}{|c|}{ (In millions of Barbados dollars) } \\
\hline Net international reserves & 518.9 & 892.5 & $1,342.0$ & $1,492.9$ & $1,845.9$ \\
\hline Central bank (net) & 612.8 & 968.8 & $1,414.1$ & $1,366.4$ & $1,501.4$ \\
\hline Commercial banks (net) & -93.9 & -76.3 & -72.1 & 126.5 & 344.5 \\
\hline Net domestic assets & $3,292.5$ & $3,303.5$ & $3,209.4$ & $3,514.2$ & $2,642.0$ \\
\hline Domestic credit & $3,696.4$ & $3,760.5$ & $3,611.9$ & $4,163.8$ & $3,532.6$ \\
\hline Public sector (net) & 324.4 & 196.6 & -63.0 & 351.4 & 481.3 \\
\hline Central government & 575.8 & 474.6 & 245.7 & 658.1 & 723.3 \\
\hline National Insurance Fund deposits & -214.8 & -241.8 & -272.0 & -284.2 & -396.3 \\
\hline Other & -36.6 & -36.3 & -36.7 & -22.5 & 154.4 \\
\hline Private sector & $3,372.0$ & $3,563.9$ & $3,674.8$ & $3,812.3$ & $3,051.3$ \\
\hline Other assets (net) & -403.9 & -457.0 & -402.4 & -649.6 & -890.5 \\
\hline Medium- and long-term foreign liabilities & 128.8 & 143.5 & 137.1 & 128.0 & 139.5 \\
\hline Liabilities to private sector & $3,682.6$ & $4,052.4$ & $4,414.3$ & $4,879.1$ & $4,348.4$ \\
\hline Money & 998.5 & $1,067.1$ & $1,148.8$ & $1,441.3$ & $1,531.0$ \\
\hline Currency in circulation & 302.7 & 310.7 & 312.4 & 337.5 & 329.0 \\
\hline Demand deposits & 695.8 & 756.4 & 836.4 & $1,103.8$ & $1,202.0$ \\
\hline Quasi-money & $1,973.2$ & $2,138.3$ & $2,237.5$ & $2,296.4$ & $2,448.7$ \\
\hline Time and savings deposits & $1,973.2$ & $2,138.3$ & $2,237.5$ & $2,296.4$ & $2,448.7$ \\
\hline \multirow[t]{2}{*}{ Other time deposits and bonds } & 710.9 & 847.0 & $1,028.1$ & $1,141.4$ & 368.8 \\
\hline & \multicolumn{5}{|c|}{ (Annual percentage changes) } \\
\hline Liabilities to private sector & 10.9 & 10.0 & 8.9 & 10.5 & -10.9 \\
\hline \multicolumn{6}{|l|}{ Of which } \\
\hline Money & 13.1 & 6.9 & 7.7 & 25.5 & 6.2 \\
\hline Currency in circulation & 12.9 & 2.6 & 0.5 & 8.0 & -2.5 \\
\hline Demand deposits & 13.2 & 8.7 & 10.6 & 32.0 & 8.9 \\
\hline \multirow[t]{2}{*}{ Quasi-money } & 10.8 & 8.4 & 4.6 & 2.6 & 6.6 \\
\hline & \multicolumn{5}{|c|}{ (In percent of GDP) } \\
\hline Liabilities to private sector & 74.2 & 78.2 & 85.9 & 93.9 & 80.7 \\
\hline \multicolumn{6}{|l|}{ Of which } \\
\hline Money & 20.1 & 20.6 & 22.3 & 27.7 & 28.4 \\
\hline Quasi-money & 39.8 & 41.3 & 43.5 & 44.2 & 45.4 \\
\hline \multirow[t]{3}{*}{ Other time deposits and bonds } & 14.3 & 16.3 & 20.0 & 22.0 & 6.8 \\
\hline & \multirow{2}{*}{\multicolumn{5}{|c|}{$\begin{array}{c}\text { (Changes in percent of beginning-of-year } \\
\text { liabilities to the private sector) }\end{array}$}} \\
\hline & & & & & \\
\hline Net domestic assets & 10.0 & 1.7 & -3.7 & 12.5 & -12.9 \\
\hline \multicolumn{6}{|l|}{ Of which } \\
\hline Public sector credit & -0.3 & -3.5 & -6.4 & 9.4 & 2.7 \\
\hline Private sector credit & 10.3 & 5.2 & 2.7 & 3.1 & -15.6 \\
\hline Money and quasi-money & 9.3 & 6.3 & 4.5 & 8.0 & 5.0 \\
\hline Money & 3.5 & 1.9 & 2.0 & 6.6 & 1.8 \\
\hline Quasi-money & 5.8 & 4.5 & 2.4 & 1.3 & 3.1 \\
\hline \multicolumn{6}{|l|}{ Memorandum items: } \\
\hline Velocity (GDP/M2) & 1.67 & 1.62 & 1.52 & 1.39 & 1.35 \\
\hline GDP (millions of Barbados dollars) & 4,963 & 5,182 & 5,140 & 5,197 & 5,391 \\
\hline
\end{tabular}

Source: Central Bank of Barbados. 
Table 16. Barbados: Summary Accounts of the Consolidated Banking System

\begin{tabular}{|c|c|c|c|c|c|}
\hline & 1999 & 2000 & 2001 & 2002 & 2003 \\
\hline & \multicolumn{5}{|c|}{ (In millions of Barbados dollars) } \\
\hline \multicolumn{6}{|c|}{ I. Banking System } \\
\hline Net international reserves & 518.9 & 892.5 & $1,342.0$ & $1,492.9$ & $1,845.9$ \\
\hline Central bank (net) & 612.8 & 968.8 & $1,414.1$ & $1,366.4$ & $1,501.4$ \\
\hline Assets & 624.8 & 968.8 & $1,414.1$ & $1,366.4$ & $1,501.4$ \\
\hline Liabilities & -12.0 & 0.0 & 0.0 & 0.0 & 0.0 \\
\hline Commercial banks (net) & -93.9 & -76.3 & -72.1 & 126.5 & 344.5 \\
\hline Net domestic assets & $2,581.6$ & $2,456.5$ & $2,181.4$ & $2,372.8$ & $2,273.2$ \\
\hline Domestic credit & $2,870.8$ & $2,819.8$ & $2,556.8$ & $3,104.1$ & $3,242.4$ \\
\hline Public sector credit (net) & 322.5 & 193.4 & -66.7 & 347.7 & 480.7 \\
\hline Central government (net) & 573.9 & 471.4 & 242.0 & 654.4 & 722.7 \\
\hline National Insurance Fund deposits 1/ & -214.8 & -241.8 & -272.0 & -284.2 & -396.3 \\
\hline Other & -36.6 & -36.3 & -36.7 & -22.5 & 154.4 \\
\hline Private sector credit & $2,455.7$ & $2,518.3$ & $2,507.6$ & $2,589.3$ & $2,612.1$ \\
\hline Credit to rest of the financial system & 92.6 & 108.1 & 115.9 & 167.1 & 149.5 \\
\hline Other items (net) & -289.2 & -363.3 & -375.4 & -731.3 & -969.1 \\
\hline Medium- and long-term foreign liabilities & 128.8 & 143.5 & 137.1 & 128.0 & 139.5 \\
\hline Broad money (M2) 2/ & $2,971.7$ & $3,205.4$ & $3,386.2$ & $3,737.7$ & $3,979.6$ \\
\hline Narrow money & 998.5 & $1,067.1$ & $1,148.8$ & $1,441.3$ & $1,531.0$ \\
\hline Currency in circulation & 302.7 & 310.7 & 312.4 & 337.5 & 329.0 \\
\hline Demand deposits & 695.8 & 756.4 & 836.4 & $1,103.8$ & $1,202.0$ \\
\hline Quasi-money & $1,973.2$ & $2,138.3$ & $2,237.5$ & $2,296.4$ & $2,448.7$ \\
\hline Time and savings deposits & $1,973.2$ & $2,138.3$ & $2,237.5$ & $2,296.4$ & $2,448.7$ \\
\hline \multicolumn{6}{|c|}{ II. Monetary Authorities } \\
\hline Net international reserves & 612.8 & 968.8 & $1,414.1$ & $1,366.4$ & $1,501.4$ \\
\hline Assets & 624.8 & 968.8 & $1,414.1$ & $1,366.4$ & $1,501.4$ \\
\hline Of which: $\mathrm{CMCF}$ & 83.5 & 74.7 & 65.9 & 57.1 & 48.4 \\
\hline Liabilities & -12.0 & 0.0 & 0.0 & 0.0 & 0.0 \\
\hline Net domestic assets & -111.5 & -405.9 & -766.7 & -458.1 & -416.0 \\
\hline Net claims on public sector & -197.2 & -466.3 & -764.6 & -476.9 & -407.4 \\
\hline Central government (net) & -193.0 & -460.5 & -757.8 & -474.4 & -405.1 \\
\hline Decentralized agencies (net) & -4.1 & -5.8 & -6.9 & -2.5 & -2.3 \\
\hline Credit to commercial banks & 15.0 & 0.0 & 0.0 & 0.0 & 0.0 \\
\hline Credit to rest of financial system & 9.0 & 9.0 & 9.0 & 9.0 & 9.0 \\
\hline Net unclassified assets & 61.7 & 51.4 & -11.1 & 9.8 & -17.6 \\
\hline Medium- and long-term foreign liabilities & 1.6 & 0.0 & 0.0 & $\mathbf{0 . 0}$ & $\mathbf{0 . 0}$ \\
\hline Monetary base & 501.3 & 562.9 & 647.4 & 908.3 & $1,085.4$ \\
\hline Liabilities to commercial banks & 198.7 & 252.2 & 335.0 & 570.8 & 756.5 \\
\hline Currency in circulation outside banks & 302.7 & 310.7 & 312.4 & 337.5 & 329.0 \\
\hline
\end{tabular}


Table 16. Barbados: Summary Accounts of the Consolidated Banking System

\begin{tabular}{|c|c|c|c|c|c|}
\hline & 1999 & 2000 & 2001 & 2002 & 2003 \\
\hline & \multicolumn{5}{|c|}{ (In millions of Barbados dollars) } \\
\hline \multicolumn{6}{|c|}{ III. Commercial Banks } \\
\hline Net international reserves & -93.9 & -76.3 & -72.1 & 126.5 & 344.5 \\
\hline Assets & 679.0 & 526.2 & 672.7 & $1,214.4$ & $1,338.6$ \\
\hline Liabilities & -772.8 & -602.5 & -744.8 & $-1,087.9$ & -994.1 \\
\hline Net claims on monetary authorities & 198.5 & 261.7 & 318.7 & 488.2 & $\mathbf{7 2 7 . 9}$ \\
\hline Net domestic assets & $2,691.6$ & $2,852.9$ & $2,964.3$ & $2,913.5$ & $2,717.8$ \\
\hline Net claims on public sector & 519.7 & 659.7 & 698.0 & 824.6 & 888.1 \\
\hline Central government (net) & 767.0 & 932.0 & 999.8 & $1,128.8$ & $1,127.8$ \\
\hline Other general government (net) & -62.8 & -85.1 & -74.1 & -78.0 & -34.8 \\
\hline Decentralized agencies & 30.3 & 54.6 & 44.3 & 58.1 & 191.5 \\
\hline National Insurance Fund deposits & -214.8 & -241.8 & -272.0 & -284.2 & -396.3 \\
\hline Credit to private sector & $2,455.7$ & $2,518.3$ & $2,507.6$ & $2,589.3$ & $2,612.1$ \\
\hline Credit to rest of financial system & 83.6 & 99.1 & 106.9 & 158.1 & 140.5 \\
\hline Liabilities to rest of financial system & 250.1 & 301.7 & 265.8 & 403.8 & 374.8 \\
\hline Net unclassified assets & -617.5 & -725.9 & -613.8 & $-1,062.2$ & $-1,297.8$ \\
\hline Medium- and long-term foreign liabilities & 127.2 & 143.5 & 137.1 & 128.0 & 139.5 \\
\hline Liabilities to monetary authorities & -15.0 & 0.0 & 0.0 & 0.0 & 0.0 \\
\hline Liabilities to private sector & $2,669.0$ & $2,894.7$ & $3,073.9$ & $3,400.2$ & $3,650.6$ \\
\hline Demand deposits & 695.8 & 756.4 & 836.4 & $1,103.8$ & $1,202.0$ \\
\hline Time and savings deposits & $1,973.2$ & $2,138.3$ & $2,237.5$ & $2,296.4$ & $2,448.7$ \\
\hline
\end{tabular}

(Changes in percent of beginning-of-year liabilities to the private sector)

Net domestic assets

$\begin{array}{rrrrr}11.1 & 6.0 & 3.8 & -1.7 & -5.8 \\ -1.2 & 5.2 & 1.3 & 4.1 & 1.9 \\ 12.9 & 2.3 & -0.4 & 2.7 & 0.7\end{array}$

Credit to public sector

Credit to private nonfinancial sector

(Annual percentage changes)

Net domestic assets

10.9

6.0

3.9

$-1.7$

$-6.7$

Of which:

Credit to public sector

Credit to private sector

\begin{tabular}{rrrrr}
-5.0 & 26.9 & 5.8 & 18.2 & 7.7 \\
14.4 & 2.6 & -0.4 & 3.3 & 0.9 \\
11.4 & 8.5 & 6.2 & 10.6 & 7.4 \\
13.2 & 8.7 & 10.6 & 32.0 & 8.9 \\
10.8 & 8.4 & 4.6 & 2.6 & 6.6 \\
\hline
\end{tabular}

Source: Central Bank of Barbados.

1/ Funds collected by the National Insurance Board, including the Transport Levy.

2/ Excludes deposits of nonbank financial institutions. 
Table 17. Barbados: Summary Accounts of the Consolidated

Nonbank Financial Intermediaries

(In millions of Barbados dollars)

\begin{tabular}{|c|c|c|c|c|c|}
\hline & 1999 & 2000 & 2001 & 2002 & 2003 \\
\hline \multicolumn{6}{|c|}{ I. Consolidated Accounts } \\
\hline Monetary reserves and currency holdings & 64.5 & 112.5 & 105.8 & 156.0 & 154.8 \\
\hline Net domestic assets & 799.3 & 927.0 & $1,057.8$ & $1,131.2$ & $1,192.9$ \\
\hline Public sector (net) & 1.9 & 3.2 & 3.7 & 3.7 & 0.6 \\
\hline Central government & 1.9 & 3.2 & 3.7 & 3.7 & 0.6 \\
\hline Other public sector & 0.0 & 0.0 & 0.0 & 0.0 & 0.0 \\
\hline Private sector & 916.3 & $1,045.6$ & $1,167.3$ & $1,223.1$ & $1,290.6$ \\
\hline Net unclassified assets & -118.9 & -121.8 & -113.2 & -95.5 & -98.2 \\
\hline Medium- and long-term foreign liabilities & 0.0 & 0.0 & 0.0 & $\mathbf{0 . 0}$ & $\mathbf{0 . 0}$ \\
\hline Liabilities to rest of financial system & 152.9 & 192.5 & 135.5 & 145.8 & 192.2 \\
\hline Liabilities to private sector & 710.9 & 847.0 & $1,028.1$ & $1,141.4$ & $1,155.6$ \\
\hline \multicolumn{6}{|c|}{ II. Trust and Mortgage Finance Companies } \\
\hline Monetary reserve and currency holdings & 14.1 & 56.0 & 21.9 & 58.6 & 51.0 \\
\hline Net domestic assets & 330.7 & 362.3 & 378.3 & 373.7 & 370.8 \\
\hline Claims on central government & 1.9 & 3.2 & 3.7 & 3.7 & 0.6 \\
\hline Credit to rest of financial system & 0.0 & 0.0 & 0.0 & 0.0 & 0.0 \\
\hline Credit to private sector & 378.6 & 423.0 & 446.4 & 442.3 & 439.1 \\
\hline Mortgage loans & 359.7 & 400.1 & 415.3 & 417.2 & 411.1 \\
\hline Residential & 308.2 & 341.4 & 354.8 & 360.0 & 365.2 \\
\hline Industrial and commercial & 50.7 & 58.1 & 59.6 & 56.3 & 44.8 \\
\hline Other & 0.7 & 0.6 & 1.0 & 0.9 & 1.1 \\
\hline Other loans & 18.9 & 22.9 & 31.0 & 25.1 & 28.0 \\
\hline Net unclassified assets & -49.8 & -63.8 & -71.8 & -72.2 & -68.8 \\
\hline Liabilities to commercial banks & 63.4 & 94.9 & 15.8 & 15.4 & 53.0 \\
\hline Liabilities to private sector & 281.4 & 323.4 & 384.4 & 417.0 & 368.8 \\
\hline Time deposits & 281.4 & 323.4 & 384.4 & 417.0 & 368.8 \\
\hline \multicolumn{6}{|c|}{ III. Barbados Mortgage Finance Company } \\
\hline Monetary reserves and currency holdings & 4.6 & 1.3 & 4.3 & 1.2 & 1.6 \\
\hline Net domestic assets & 82.5 & 95.3 & 115.1 & 128.4 & 136.8 \\
\hline Credit to private sector & 110.9 & 127.2 & 147.8 & 165.1 & 176.4 \\
\hline Net unclassified assets & -28.4 & -31.9 & -32.7 & -36.6 & -39.7 \\
\hline Liabilities to rest of financial system & 87.1 & 96.6 & 119.4 & 129.7 & 138.4 \\
\hline Trust companies & 0.0 & 0.0 & 0.0 & 0.0 & 0.0 \\
\hline Banks and other financial institutions & 87.1 & 96.6 & 119.4 & 129.7 & 138.4 \\
\hline
\end{tabular}


Table 17. Barbados: Summary Accounts of the Consolidated Nonbank Financial Intermediaries

(In millions of Barbados dollars)

\begin{tabular}{|c|c|c|c|c|c|}
\hline & 1999 & 2000 & 2001 & 2002 & 2003 \\
\hline \multicolumn{6}{|c|}{ IV. Finance Companies and Merchant Banks } \\
\hline Monetary reserves and currency holdings & 7.3 & 16.1 & 27.2 & 24.4 & 24.4 \\
\hline Net domestic assets & 127.6 & 162.7 & 208.7 & 227.2 & 236.5 \\
\hline Credit to private sector & 127.2 & 143.9 & 173 & 163.2 & 169.9 \\
\hline Net unclassified assets & 8.4 & 26.9 & 44.3 & 64.0 & 66.6 \\
\hline Liabilities to rest of financial system & 2.4 & 1.0 & 0.3 & 0.8 & 0.8 \\
\hline Liabilities to private sector & 132.5 & 177.8 & 235.6 & 250.9 & 260.2 \\
\hline Time deposits & 132.5 & 177.8 & 235.6 & 250.9 & 260.2 \\
\hline \multicolumn{6}{|c|}{ V. Credit Unions } \\
\hline Monetary reserves and currency holdings & 38.5 & 39.1 & 52.4 & 71.7 & 77.8 \\
\hline Net domestic assets & 258.5 & 306.7 & 355.8 & 401.8 & 448.8 \\
\hline Credit to private sector & 299.6 & 351.5 & 400.1 & 452.5 & 505.1 \\
\hline Loans & 265.2 & 302.8 & 349.4 & 387.0 & 430.9 \\
\hline Investments & 34.4 & 48.7 & 50.7 & 65.5 & 74.2 \\
\hline Net unclassified assets & -41.1 & -44.8 & -44.4 & -50.6 & -56.3 \\
\hline Liabilities to the private sector & 297.0 & 345.8 & 408.1 & 473.5 & 526.6 \\
\hline Deposits & 71.8 & 87.3 & 113.2 & 138.1 & 154.0 \\
\hline Shares & 225.2 & 258.5 & 294.9 & 335.4 & 372.6 \\
\hline
\end{tabular}

Source: Central Bank of Barbados. 
Table 18. Barbados: Financial System Credit to the Private Sector (In millions of Barbados dollars)

\begin{tabular}{lrrrrr}
\hline & 1999 & 2000 & 2001 & 2002 & 2003 \\
\hline & & & & & \\
Total credit to private sector & $\mathbf{3 , 3 7 2 . 0}$ & $\mathbf{3 , 5 6 3 . 9}$ & $\mathbf{3 , 6 7 4 . 8}$ & $\mathbf{3 , 8 1 2 . 3}$ & $\mathbf{3 , 9 0 2 . 7}$ \\
& & & & $\mathbf{2 , 6 1 2 . 1}$ \\
Commercial banks & $\mathbf{2 , 4 5 5 . 7}$ & $\mathbf{2 , 5 1 8 . 3}$ & $\mathbf{2 , 5 0 7 . 6}$ & $\mathbf{2 , 5 8 9 . 3}$ & $\mathbf{2 , 5}$ \\
Agriculture and fisheries & 50.7 & 69.3 & 56.9 & 48.0 & 43.8 \\
Manufacturing & 96.6 & 88.0 & 92.6 & 86.1 & 99.1 \\
Distributive trades & 321.4 & 275.7 & 251.0 & 289.0 & 276.9 \\
Construction & 182.0 & 233.5 & 228.2 & 227.6 & 207.6 \\
Mortgages & 254.7 & 287.7 & 366.1 & 457.4 & 509.9 \\
Tourism & 302.2 & 328.6 & 293.7 & 336.0 & 381.9 \\
Professional and other services & 202.0 & 174.0 & 179.9 & 175.7 & 181.3 \\
Other (including personal) & $1,046.1$ & $1,061.5$ & $1,039.1$ & 969.4 & 911.7 \\
& & & & $\mathbf{1}$ \\
Nonbank financial institutions & $\mathbf{9 1 6 . 3}$ & $\mathbf{1 , 0 4 5 . 6}$ & $\mathbf{1 , 1 6 7 . 3}$ & $\mathbf{1 , 2 2 3 . 1}$ & $\mathbf{1 , 2 9 0 . 6}$ \\
Mortgages & 470.6 & 527.3 & 563.1 & 582.3 & 587.6 \\
Other & 445.7 & 518.3 & 604.1 & 640.8 & 703.0 \\
& & & & &
\end{tabular}

Source: Central Bank of Barbados. 
Table 19. Barbados: Liquidity Position of Commercial Banks (In millions of Barbados dollars)

\begin{tabular}{|c|c|c|c|c|c|}
\hline & 1999 & 2000 & 2001 & 2002 & 2003 \\
\hline \multicolumn{6}{|l|}{ Cash reserve requirement } \\
\hline Required reserves & 200 & 220 & 196 & 214 & 237 \\
\hline Actual reserves & 188 & 234 & 292 & 487 & 692 \\
\hline Cash in vaults & 93 & 85 & 78 & 92 & 144 \\
\hline Deposits with central bank & 96 & 149 & 214 & 395 & 548 \\
\hline Excess reserves & -12 & 14 & 96 & 273 & 455 \\
\hline \multicolumn{6}{|c|}{ Government securities requirement } \\
\hline Required holdings $1 /$ & 673 & 701 & 783 & 692 & 763 \\
\hline Actual holdings & 805 & 970 & 1,050 & 1,188 & 1,307 \\
\hline Treasury bills & 355 & 488 & 471 & 492 & 568 \\
\hline Debentures 2/ & 393 & 455 & $\ldots$ & $\ldots$ & $\ldots$ \\
\hline Other securities & 57 & 27 & 579 & 697 & 739 \\
\hline Excess holdings & 132 & 269 & 268 & 496 & 543 \\
\hline \multicolumn{6}{|l|}{ Cash reserve requirement 3 / } \\
\hline Required reserves & 6 & 6 & 5 & 5 & 5 \\
\hline Actual reserves & 6 & 6 & 7 & 11 & 15 \\
\hline Excess reserves & 0 & 0 & 2 & 6 & 10 \\
\hline \multicolumn{6}{|l|}{ Memorandum item: } \\
\hline Cash reserves deposit base $3 /$ & 3,332 & 3,674 & 3,926 & 4,286 & 4,735 \\
\hline \multicolumn{6}{|c|}{ Government securities requirement 4/ } \\
\hline Required holdings $1 /$ & 20 & 19 & 19 & 16 & 16 \\
\hline Actual holdings & 24 & 26 & 25 & 27 & 27 \\
\hline Excess holdings & 4 & 7 & 6 & 11 & 11 \\
\hline \multicolumn{6}{|l|}{ Memorandum item: } \\
\hline Securities deposit base 4/ & 3,363 & 3,689 & 4,119 & 4,327 & 4,771 \\
\hline
\end{tabular}

Source: Central Bank of Barbados.

1/ Eligible securities include Treasury bills and debentures, and securities issued by the statutory bodies.

2/ From February 01, 2001, the central bank removed the stipulation which required commercial banks to hold a specified amount of their deposits in debentures.

3/ As a percentage of the cash reserves deposit base, calculated as the average of three consecutive end-Wednesday deposits.

4/ As a percentage of the securities deposit base-end-month stocks. 
Table 20. Barbados: Selected Interest Rates

(In percent per annum: period averages)

\begin{tabular}{|c|c|c|c|c|c|c|c|c|c|c|c|}
\hline \multirow[b]{2}{*}{ Period } & \multirow[b]{2}{*}{$\begin{array}{r}\text { Central Bank } \\
\text { iscount }\end{array}$} & \multicolumn{2}{|c|}{ Average Lending Rates } & \multirow{2}{*}{$\begin{array}{r}\text { Weighted } \\
\text { Average } \\
\text { Deposit } \\
\text { Rate }\end{array}$} & \multirow[b]{2}{*}{$\begin{array}{c}\text { Savings } \\
\text { Deposits }\end{array}$} & \multirow[b]{2}{*}{$\begin{array}{r}\text { Three-Month } \\
\text { Deposits }\end{array}$} & \multirow{2}{*}{$\begin{array}{l}\text { Average } \\
\text { Treasury } \\
\text { Bill Rate }\end{array}$} & \multirow{2}{*}{$\begin{array}{r}\text { Change in } \\
\text { Retail Price } \\
\text { Index }\end{array}$} & \multicolumn{3}{|c|}{ Memo: U.S. Interest Rates } \\
\hline & & $\begin{array}{r}\text { Selected } \\
\text { Loans }\end{array}$ & $\begin{array}{r}\text { All } \\
\text { Loans }\end{array}$ & & & & & & $\begin{array}{r}\text { Prime } \\
\text { Lending }\end{array}$ & $\begin{array}{c}\text { Three-month } \\
\text { Deposits }\end{array}$ & $\begin{array}{r}\text { Treasury Bil } \\
\text { Rate }\end{array}$ \\
\hline 1993 & 8.0 & 10.6 & 11.3 & 4.8 & 5.0 & 2.9 & 7.2 & 1.1 & 6.0 & 3.2 & 3.0 \\
\hline 1994 & 9.5 & 11.0 & 11.9 & 5.0 & 5.0 & 5.0 & 7.8 & 0.7 & 7.1 & 4.6 & 4.3 \\
\hline 1995 & 12.5 & 10.9 & 11.8 & 5.2 & 5.0 & 5.0 & 8.3 & 1.9 & 8.8 & 5.9 & 5.5 \\
\hline 1996 & 12.5 & 11.1 & 11.9 & 5.2 & 5.0 & 5.0 & 6.6 & 2.4 & 8.3 & 5.4 & 5.0 \\
\hline 1997 & 9.9 & 10.6 & 11.6 & 4.5 & 4.3 & 4.4 & 3.5 & 7.7 & 8.5 & 5.6 & 5.1 \\
\hline 1998 & 9.0 & 10.4 & 11.1 & 4.2 & 4.0 & 4.5 & 5.7 & -1.3 & 8.4 & 5.5 & 4.8 \\
\hline 1999 & 10.0 & 10.5 & 11.1 & 4.3 & 4.3 & 5.1 & 5.8 & 1.6 & 8.0 & 5.4 & 4.7 \\
\hline 2000 & 10.0 & 11.3 & 11.8 & 4.9 & 4.5 & 2.5 & 3.9 & 2.4 & 9.5 & 6.5 & 6.0 \\
\hline 2001 & 7.5 & 9.3 & 11.1 & 3.1 & 3.3 & 3.8 & 2.0 & 2.8 & 5.2 & 2.1 & 2.0 \\
\hline 2002 & 7.5 & 8.3 & 10.7 & 2.7 & 3.1 & 2.6 & 2.0 & 0.2 & 4.7 & 1.7 & 1.6 \\
\hline 1998 & & & & & & & & & & & \\
\hline I & 9.0 & 10.4 & 11.1 & 4.2 & 4.0 & 4.5 & 5.6 & 0.1 & 8.5 & 5.6 & 5.1 \\
\hline II & 9.0 & 10.4 & 11.1 & 4.2 & 4.0 & 4.5 & 5.7 & 1.3 & 8.5 & 5.6 & 5.0 \\
\hline III & 9.0 & 10.4 & 11.1 & 4.2 & 4.0 & 4.5 & 5.6 & -0.2 & 8.5 & 5.5 & 4.9 \\
\hline IV & 9.0 & 10.4 & 11.1 & 4.2 & 4.0 & 4.5 & 5.7 & 0.5 & 7.9 & 5.2 & 4.3 \\
\hline 1999 & & & & & & & & & & & \\
\hline I & 9.0 & 10.4 & 11.1 & 4.2 & 4.0 & 5.0 & 5.6 & -1.2 & 7.8 & 4.9 & 4.4 \\
\hline II & 10.0 & 10.4 & 11.1 & 4.2 & 4.0 & 5.0 & 5.9 & 1.5 & 7.8 & 5.0 & 4.5 \\
\hline III & 10.0 & 10.4 & 11.1 & 4.2 & 4.0 & 5.0 & 5.9 & 3.0 & 8.1 & 5.4 & 4.7 \\
\hline IV & 10.0 & 10.7 & 11.0 & 4.7 & 5.0 & 5.3 & 6.1 & -0.3 & 8.4 & 6.1 & 5.1 \\
\hline 2000 & & & & & & & & & & & \\
\hline I & 10.0 & 11.3 & 12.2 & 5.0 & 5.0 & 5.5 & 6.1 & -1.7 & 8.7 & 6.0 & 5.5 \\
\hline II & 10.0 & 11.5 & 12.2 & 5.0 & 5.0 & 5.5 & 5.7 & 0.8 & 9.3 & 6.6 & 5.8 \\
\hline III & 10.0 & 11.5 & 12.2 & 4.9 & 4.5 & 5.3 & 4.5 & 3.1 & 9.5 & 6.6 & 6.0 \\
\hline IV & 10.0 & 11.3 & 11.9 & 4.9 & 4.5 & 4.8 & 3.9 & 1.6 & 9.5 & 6.6 & 6.0 \\
\hline 2001 & & & & & & & & & & & \\
\hline I & 10.0 & 11.6 & 11.9 & 4.6 & 4.9 & 4.6 & 3.7 & -2.2 & 8.6 & 5.3 & 4.9 \\
\hline II & 9.5 & 11.1 & 11.6 & 4.3 & 4.4 & 4.3 & 3.6 & 2.5 & 7.3 & 4.1 & 3.7 \\
\hline III & 8.5 & 10.2 & 11.4 & 3.7 & 3.8 & 3.8 & 2.7 & -0.2 & 6.6 & 3.3 & 3.3 \\
\hline IV & 8.5 & 9.3 & 11.1 & 3.1 & 3.3 & 3.8 & 2.0 & -0.9 & 5.2 & 2.1 & 2.0 \\
\hline 2002 & & & & & & & & & & & \\
\hline I & 7.5 & 8.7 & 10.9 & 2.7 & 3.3 & 2.5 & 1.2 & 2.4 & 4.8 & 1.9 & 1.7 \\
\hline II & 7.5 & 8.4 & 10.7 & 2.7 & 3.3 & 2.5 & 2.8 & 1.7 & 4.8 & 1.8 & 1.7 \\
\hline III & 7.5 & 8.3 & 10.8 & 2.6 & 3.3 & 3.0 & 2.5 & 0.3 & 4.8 & 1.8 & 1.7 \\
\hline IV & 7.5 & 7.9 & 10.4 & 2.6 & 2.5 & 2.5 & 1.5 & 0.1 & 4.5 & 1.3 & 1.4 \\
\hline 2003 & & & & & & & & & & & \\
\hline I & 7.5 & 8.2 & 10.3 & 2.6 & 2.8 & 2.5 & 2.5 & 0.1 & 4.3 & 1.3 & 1.2 \\
\hline II & 7.5 & 8.2 & 10.2 & 2.6 & 2.8 & 2.5 & 1.9 & 0.4 & 4.2 & 1.2 & 1.1 \\
\hline III & 7.5 & 8.4 & 10.2 & 2.6 & 2.8 & 2.5 & 0.9 & 1.2 & 4.0 & 1.1 & 0.9 \\
\hline IV & 7.5 & 8.4 & 10.1 & 2.6 & 2.8 & 2.5 & 0.8 & 1.5 & 4.0 & 1.1 & 0.9 \\
\hline
\end{tabular}

Sources: Central Bank of Barbados, Economic and Financial Statistics; and IMF, International Financial Statistics. 
Table 21. Barbados: Summary Accounts of Offshore Banks

(In millions of Barbados dollars)

\begin{tabular}{|c|c|c|c|c|c|}
\hline & 1999 & 2000 & 2001 & 2002 & $\begin{array}{l}\text { Sept. } \\
2003\end{array}$ \\
\hline \multicolumn{6}{|c|}{ I. Balance Sheet (end of period) } \\
\hline Assets & 40,790 & 43,785 & 57,260 & 65,551 & $\mathbf{5 9 , 8 6 7}$ \\
\hline Due from financial institutions & 17,966 & 18,335 & 27,874 & 31,469 & 28,480 \\
\hline Other banks & 2,390 & 1,842 & 2,450 & 2,616 & 2,426 \\
\hline In Barbados & 413 & 616 & 661 & 1,079 & 1,110 \\
\hline Abroad & 1,977 & 1,226 & 1,790 & 1,537 & 1,315 \\
\hline Head office and branches abroad & 15,576 & 16,494 & 25,424 & 28,847 & 26,048 \\
\hline Loans and advances to nonresidents & 1,566 & 1,664 & 2,034 & 3,193 & $\mathbf{3 , 5 5 8}$ \\
\hline Investments abroad & 20,351 & 22,418 & 25,942 & 30,052 & 26,747 \\
\hline Unclassified assets & 907 & 1,367 & 1,411 & 838 & $\mathbf{1 , 0 8 7}$ \\
\hline Liabilities & 40,790 & 43,785 & 57,260 & 65,551 & $\mathbf{5 9 , 8 6 7}$ \\
\hline Deposits & 2,508 & 2,255 & 2,688 & 2,842 & 2,364 \\
\hline Demand & 776 & 754 & 818 & 865 & 702 \\
\hline Time and saving & 1,732 & 1,502 & 1,870 & 1,977 & 1,662 \\
\hline Due to head offices and branches abroad & 16,367 & 11,869 & 13,137 & 16,497 & 18,181 \\
\hline Due to other financial institutions & 85 & 374 & 753 & 928 & 384 \\
\hline Capital and reserves & 20,726 & 22,931 & 27,272 & 29,234 & 29,192 \\
\hline Unclassified liabilities & $1,103.6$ & $6,355.5$ & $13,410.7$ & $16,050.0$ & $\mathbf{9 , 7 4 6 . 2}$ \\
\hline \multicolumn{6}{|c|}{ II. Profits and Loss (flow during period) } \\
\hline Earnings & 3,350 & 3,985 & 3,740 & 2,455 & 2,412 \\
\hline Interest on loans and discounts & 242 & 295 & 253 & 230 & 256 \\
\hline Other (mainly investment) income & 3,108 & 3,690 & 3,487 & 2,224 & 2,156 \\
\hline Expenses & 1,967 & 3,619 & 1,807 & 2,955 & 1,741 \\
\hline Interest paid on deposits & 139 & 171 & 193 & 136 & 78 \\
\hline Interest on borrowings & 659 & 560 & 641 & 581 & 337 \\
\hline Other expenses & 1,168 & 2,889 & 973 & 2,238 & 1,326 \\
\hline Net operating surplus & 1,383 & 366 & 1,933 & -501 & 672 \\
\hline
\end{tabular}

Source: Central Bank of Barbados. 
Table 22. Barbados: Summary Balance of Payments

(In millions of U.S. dollars)

\begin{tabular}{|c|c|c|c|c|c|}
\hline & 1999 & 2000 & 2001 & 2002 & 2003 \\
\hline Current account & -149 & -145 & -94 & -171 & -210 \\
\hline Merchandise trade balance & -716 & -744 & -681 & -702 & -797 \\
\hline Exports & 274 & 286 & 271 & 251 & 229 \\
\hline \multicolumn{6}{|l|}{ Of which: } \\
\hline Domestic exports & 196 & 190 & 176 & 167 & 163 \\
\hline Sugar and molasses & 28 & 26 & 22 & 19 & 21 \\
\hline Other & 168 & 164 & 154 & 148 & 143 \\
\hline Imports & 989 & 1030 & 952 & 953 & 1025 \\
\hline Services (net) & 571 & 603 & 587 & 546 & 584 \\
\hline Travel (credit) & 687 & 723 & 714 & 658 & 698 \\
\hline Investment income (net) & -71 & -82 & -93 & -102 & -96 \\
\hline \multicolumn{6}{|l|}{ Of which: } \\
\hline Interest on public debt & -29 & -36 & -45 & -52 & -52 \\
\hline Current transfers (net) & 67 & 78 & 94 & 86 & 99 \\
\hline Capital account and finincial account & 144 & 324 & 317 & 147 & 278 \\
\hline Long-term capital & 111 & 287 & 277 & 13 & 102 \\
\hline Public sector & 56 & 123 & 152 & 0 & -15 \\
\hline Central government & 43 & 104 & 165 & 2 & 3 \\
\hline Government guaranteed & 14 & 20 & -12 & -2 & -19 \\
\hline Private sector & 55 & 164 & 125 & 13 & 117 \\
\hline Direct investment & 54 & 156 & 128 & 17 & 121 \\
\hline Commercial banks & 1 & 8 & -3 & -4 & -4 \\
\hline Short-term capital & 33 & 37 & 40 & 134 & 176 \\
\hline Commercial banks & 14 & 9 & 2 & 0 & 0 \\
\hline Other private (including errors and ommissions) & 61 & 27 & 37 & 134 & 176 \\
\hline Overall balance (deficit -) & 37 & 178 & 223 & -24 & 67 \\
\hline \multicolumn{6}{|l|}{ Memorandum items: } \\
\hline Current account (percent of GDP) & -6.0 & -5.6 & -3.6 & -6.4 & -7.8 \\
\hline Gross international reserves (in millions of U.S. dollars) & 312 & 484 & 707 & 683 & 751 \\
\hline Gross international reserves (in months of imports) & 2.5 & 3.7 & 5.7 & 5.8 & 5.9 \\
\hline Public external debt (in millions of U.S. dollars) & 463.5 & 582.5 & 742.5 & 707 & 711.5 \\
\hline Public external debt (percent of GDP) & 18.7 & 22.5 & 28.9 & 27.2 & 26.4 \\
\hline
\end{tabular}

Sources: Central Bank of Barbados; Barbados Statistical Service; and Fund staff estimates. 
Table 23. Barbados: Total Exports

\begin{tabular}{|c|c|c|c|c|c|}
\hline & 1999 & 2000 & 2001 & 2002 & $\begin{array}{l}\text { Prel. } \\
2003\end{array}$ \\
\hline & \multicolumn{5}{|c|}{ (In millions of U.S. dollars) } \\
\hline $\begin{array}{l}\text { Total exports } \\
\text { Of which: }\end{array}$ & 274 & 286 & 271 & 251 & 229 \\
\hline Sugar and molasses & 28 & 27 & 22 & 19 & 21 \\
\hline Rum & 14 & 13 & 18 & 18 & 16 \\
\hline Margarine and lard & 5 & 4 & 5 & 5 & 4 \\
\hline Other food and beverages & 25 & 26 & 20 & 26 & 17 \\
\hline Chemicals & 26 & 22 & 19 & 21 & 19 \\
\hline Electronic components & 28 & 22 & 19 & 15 & 12 \\
\hline Clothing & 3 & 3 & 2 & 1 & 1 \\
\hline Other manufactures & 69 & 74 & 70 & 63 & 39 \\
\hline Other exports (including re-exports) & 76 & 96 & 96 & 84 & 24 \\
\hline \multicolumn{6}{|c|}{ (In percent of GDP) } \\
\hline \multicolumn{6}{|l|}{ Memorandum items: } \\
\hline & 11.0 & 11.0 & 10.6 & 9.7 & 8.5 \\
\hline & \multicolumn{5}{|c|}{ (Annual changes, in percent) } \\
\hline Sugar and molasses & 1.4 & -4.6 & -17.6 & -14.1 & 8.5 \\
\hline Chemicals & 2.0 & -16.9 & -11.1 & 9.3 & -10.0 \\
\hline Electronic components & 10.2 & -20.3 & -14.7 & -24.1 & -17.2 \\
\hline Other manufactures & 12.4 & 7.7 & -5.7 & -9.9 & -38.2 \\
\hline Others & 14.1 & 26.0 & 0.4 & -12.5 & -72.0 \\
\hline
\end{tabular}

Sources: Central Bank of Barbados; Barbados Statistical Service; and Fund staff estimates. 
Table 24. Barbados: Total Imports

\begin{tabular}{|c|c|c|c|c|c|}
\hline & 1999 & 2000 & 2001 & 2002 & $\begin{array}{l}\text { Prel. } \\
2003\end{array}$ \\
\hline & \multicolumn{5}{|c|}{ (In millions of U.S. dollars) } \\
\hline $\begin{array}{l}\text { Total imports } \\
\text { of which: }\end{array}$ & 989 & 1,030 & 952 & 953 & 1,025 \\
\hline Consumer goods & 437 & 441 & 421 & 424 & 408 \\
\hline Nondurables & 251 & 247 & 255 & 267 & 249 \\
\hline Food and beverages & 159 & 159 & 165 & 168 & 159 \\
\hline Other & 92 & 87 & 91 & 99 & 91 \\
\hline Durables & 102 & 101 & 81 & 77 & 80 \\
\hline Motorcars & 60 & 47 & 35 & 33 & 37 \\
\hline Other & 42 & 53 & 46 & 44 & 43 \\
\hline Other manufactured goods & 84 & 93 & 85 & 80 & 80 \\
\hline Intermediate goods & 363 & 406 & 355 & 366 & 373 \\
\hline Fuels & 68 & 89 & 81 & 95 & 114 \\
\hline Chemicals & 53 & 52 & 54 & 53 & 52 \\
\hline Textiles & 18 & 20 & 18 & 17 & 17 \\
\hline Foods and fats & 26 & 21 & 21 & 22 & 20 \\
\hline Electrical components & 29 & 32 & 26 & 23 & 24 \\
\hline Other manufactured goods & 82 & 88 & 80 & 78 & 74 \\
\hline Construction materials & 87 & 104 & 75 & 78 & 74 \\
\hline Capital goods & 239 & 224 & 206 & 202 & 214 \\
\hline \multirow[t]{2}{*}{ Unclassified goods } & 3 & 3 & 3 & 4 & 3 \\
\hline & \multicolumn{5}{|c|}{ (In percent of GDP) } \\
\hline \multicolumn{6}{|l|}{ Memorandum items: } \\
\hline Total imports & 39.9 & 39.8 & 37.1 & 36.7 & 38.0 \\
\hline Consumer goods & 17.6 & 17.0 & 16.4 & 16.3 & 15.1 \\
\hline Intermediate goods & 14.6 & 15.7 & 13.8 & 14.1 & 13.8 \\
\hline \multirow[t]{2}{*}{ Capital goods } & 9.6 & 8.6 & 8.0 & 7.8 & 7.9 \\
\hline & \multicolumn{5}{|c|}{ (Annual changes, in percent) } \\
\hline \multicolumn{6}{|l|}{ Import growth } \\
\hline Total imports & 9.8 & 4.1 & -7.6 & 0.1 & 7.6 \\
\hline Consumer goods & 7.2 & 0.9 & -4.4 & 0.6 & -3.7 \\
\hline Intermediate goods & 8.8 & 11.8 & -12.5 & 3.1 & 1.8 \\
\hline Capital goods & 15.7 & -6.4 & -8.0 & -1.7 & 5.6 \\
\hline
\end{tabular}

Sources: Central Bank of Barbados; Barbados Statistical Service; and Fund staff estimates. 
Table 25. Barbados: Services, Investment Income, and Current Transfers

\begin{tabular}{|c|c|c|c|c|c|}
\hline & 1999 & 2000 & 2001 & 2002 & 2003 \\
\hline & \multicolumn{4}{|c|}{ (In millions of U.S. dollars) } & \\
\hline Services (net) & 571 & 603 & 587 & 546 & 584 \\
\hline Travel & 600 & 629 & 596 & 559 & 675 \\
\hline Credit & 687 & 723 & 697 & 658 & 698 \\
\hline Debit & 87 & 94 & 101 & 99 & 23 \\
\hline Transportation & -134 & -139 & -134 & -134 & -142 \\
\hline Credit & 23 & 25 & 22 & 21 & 20 \\
\hline Debit & 157 & 164 & 156 & 155 & 162 \\
\hline Government & 7 & 8 & 8 & 12 & 11 \\
\hline Credit & 32 & 35 & 36 & 38 & 40 \\
\hline Debit & 25 & 27 & 28 & 26 & 29 \\
\hline Other services & 98 & 105 & 99 & 110 & 118 \\
\hline Credit & 287 & 308 & 313 & 324 & 331 \\
\hline Debit & 189 & 202 & 214 & 215 & 214 \\
\hline Income & -71 & -82 & -93 & -102 & -97 \\
\hline Credit & 67 & 70 & 73 & 72 & 72 \\
\hline Debit & 138 & 152 & 166 & 174 & 168 \\
\hline \multirow[t]{2}{*}{ Current transfers } & 67 & 78 & 94 & 86 & 99 \\
\hline & \multicolumn{4}{|c|}{ (In percent of GDP) } & \\
\hline \multicolumn{6}{|l|}{ Memorandum item: } \\
\hline Gross travel receipts & 27.7 & 27.9 & 27.1 & 25.3 & 25.9 \\
\hline
\end{tabular}

Sources: Central Bank of Barbados; and Fund staff estimates. 
Table 26. Barbados: Direction of Trade 1/

\begin{tabular}{|c|c|c|c|c|c|}
\hline & 1999 & 2000 & 2001 & 2002 & $\begin{array}{l}\text { Prel. } \\
2003\end{array}$ \\
\hline & \multicolumn{5}{|c|}{ (In millions of U.S. dollars) } \\
\hline Total exports, f.o.b. & 274 & 286 & 271 & 251 & 229 \\
\hline United States 2/ & 43 & 42 & 37 & 32 & 34 \\
\hline CARICOM & 114 & 114 & 103 & 99 & 101 \\
\hline United Kingdom & 35 & 35 & 30 & 26 & 28 \\
\hline Canada & 7 & 6 & 7 & 5 & 4 \\
\hline Rest of Europe 3/ & 9 & 8 & 10 & 11 & 9 \\
\hline Japan & 0.3 & 0.3 & 0.2 & 0.2 & 0.2 \\
\hline Other & 66 & 81 & 84 & 78 & 53 \\
\hline Total imports, c.i.f. & 989 & 1,030 & 952 & 953 & 1,025 \\
\hline United States 2/ & 443 & 472 & 439 & 426 & 445 \\
\hline CARICOM & 191 & 225 & 210 & 218 & 240 \\
\hline United Kingdom & 92 & 93 & 86 & 79 & 75 \\
\hline Canada & 46 & 48 & 40 & 37 & 43 \\
\hline Rest of Europe 3/ & 85 & 84 & 92 & 94 & 124 \\
\hline Japan & 84 & 60 & 45 & 45 & 54 \\
\hline \multirow[t]{2}{*}{ Other } & 48 & 48 & 41 & 54 & 45 \\
\hline & \multicolumn{5}{|c|}{ (In percent of total) } \\
\hline Total exports, f.o.b. & 100 & 100 & 100 & 100 & 100 \\
\hline United States 2/ & 15.5 & 14.7 & 13.6 & 12.6 & 14.7 \\
\hline CARICOM & 41.7 & 40.0 & 38.0 & 39.6 & 44.2 \\
\hline United Kingdom & 12.6 & 12.4 & 11.2 & 10.2 & 12.0 \\
\hline Canada & 2.5 & 1.9 & 2.6 & 1.8 & 1.7 \\
\hline Rest of Europe 3/ & 3.3 & 2.7 & 3.7 & 4.5 & 3.9 \\
\hline Japan & 0.1 & 0.1 & 0.1 & 0.1 & 0.1 \\
\hline Other & 24.2 & 28.2 & 30.9 & 31.2 & 23.3 \\
\hline Total imports, c.i.f. & 100 & 100 & 100 & 100 & 100 \\
\hline United States 2/ & 44.8 & 45.8 & 46.1 & 44.6 & 43.4 \\
\hline CARICOM & 19.3 & 21.8 & 22.0 & 22.8 & 23.4 \\
\hline United Kingdom & 9.3 & 9.1 & 9.0 & 8.3 & 7.3 \\
\hline Canada & 4.7 & 4.7 & 4.2 & 3.9 & 4.1 \\
\hline Rest of Europe 3/ & 8.6 & 8.1 & 9.7 & 9.9 & 12.1 \\
\hline Japan & 8.5 & 5.8 & 4.7 & 4.7 & 5.2 \\
\hline Other & 4.9 & 4.6 & 4.3 & 5.7 & 4.4 \\
\hline
\end{tabular}

Sources: Barbados Statistical Services; Central Bank of Barbados; and Fund staff estimates.

$1 /$ Includes re-exports.

2/ Includes Puerto Rico.

3/ Includes the 15 EU member states, except for the United Kingdom. 


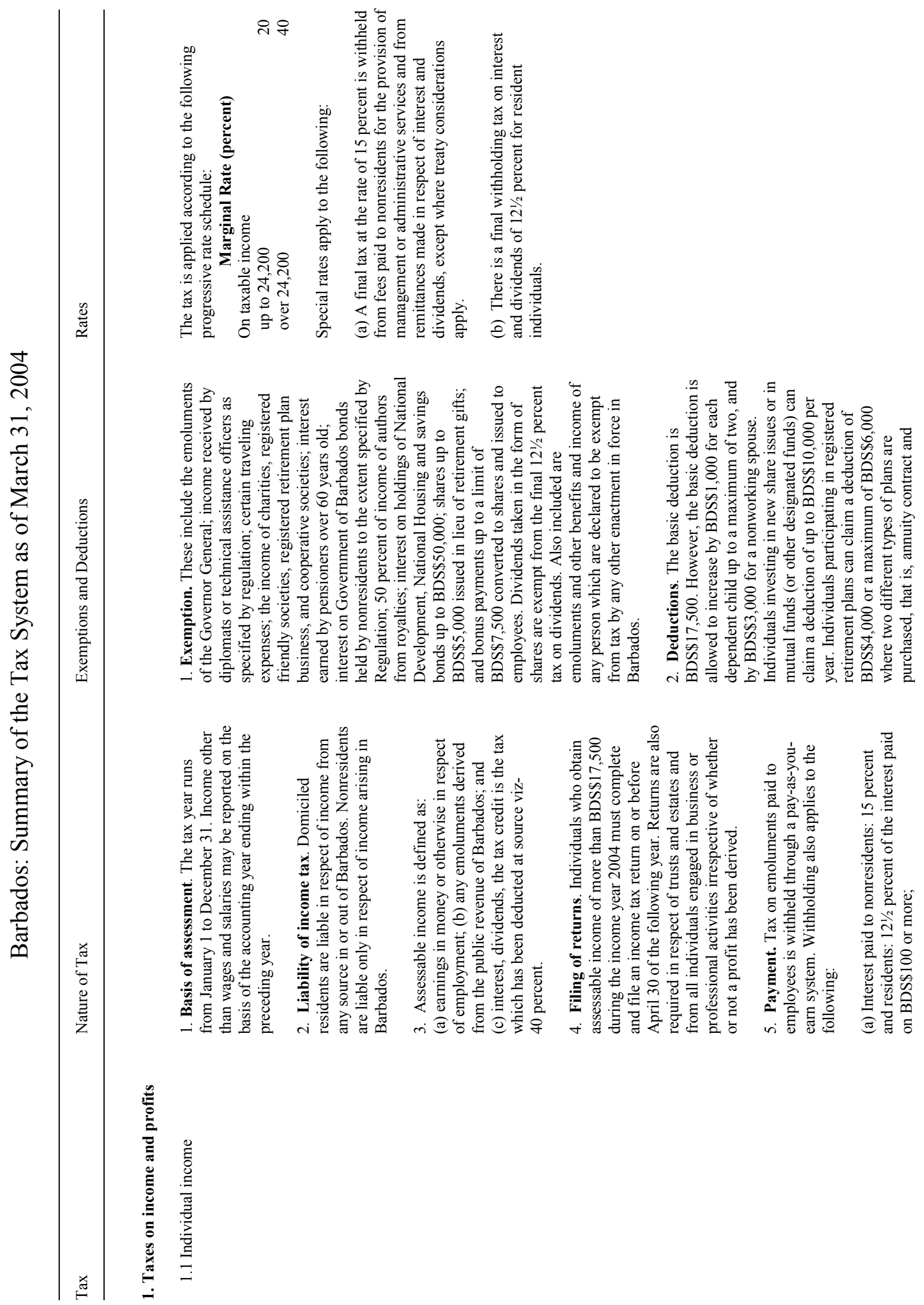




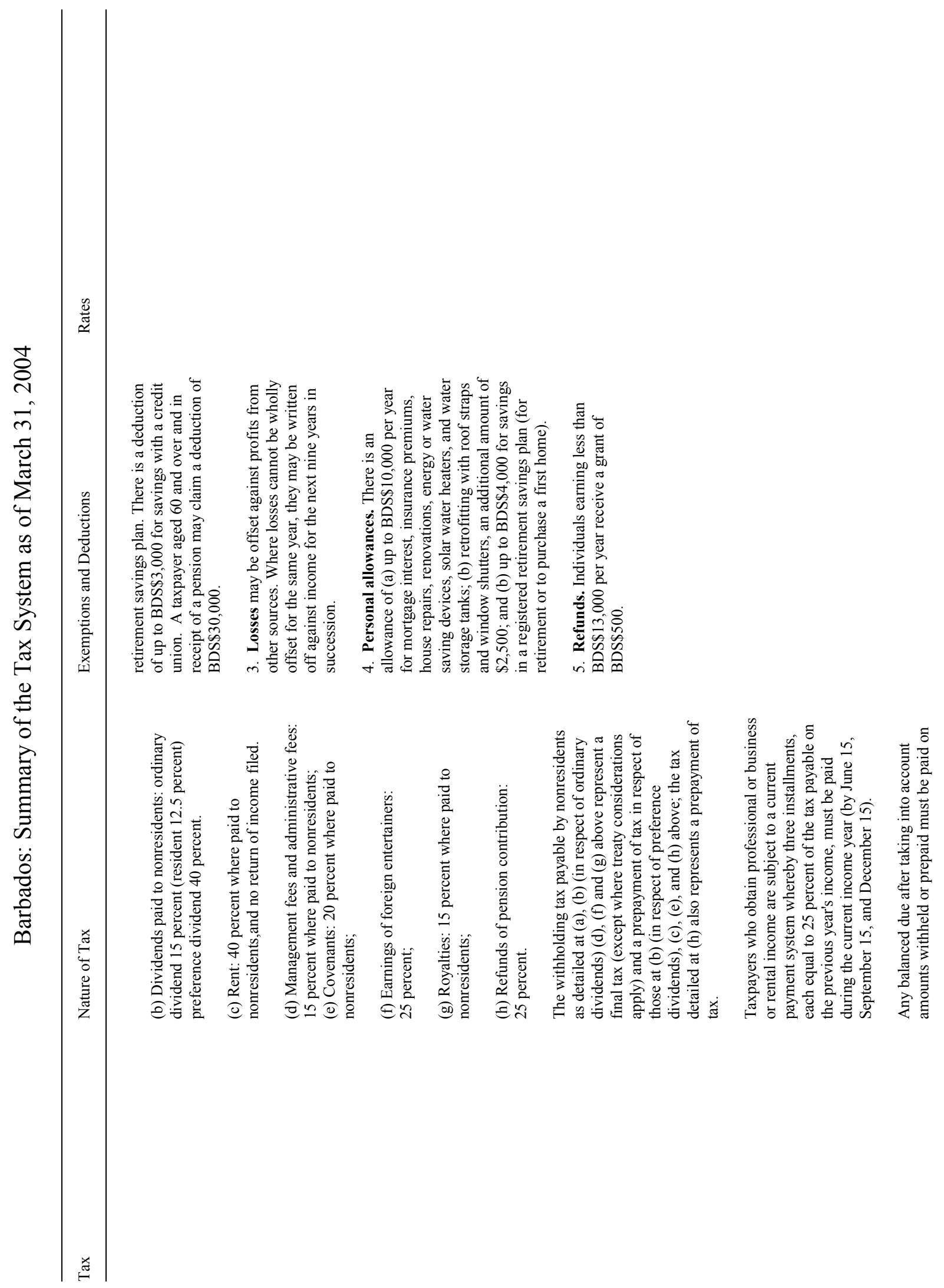




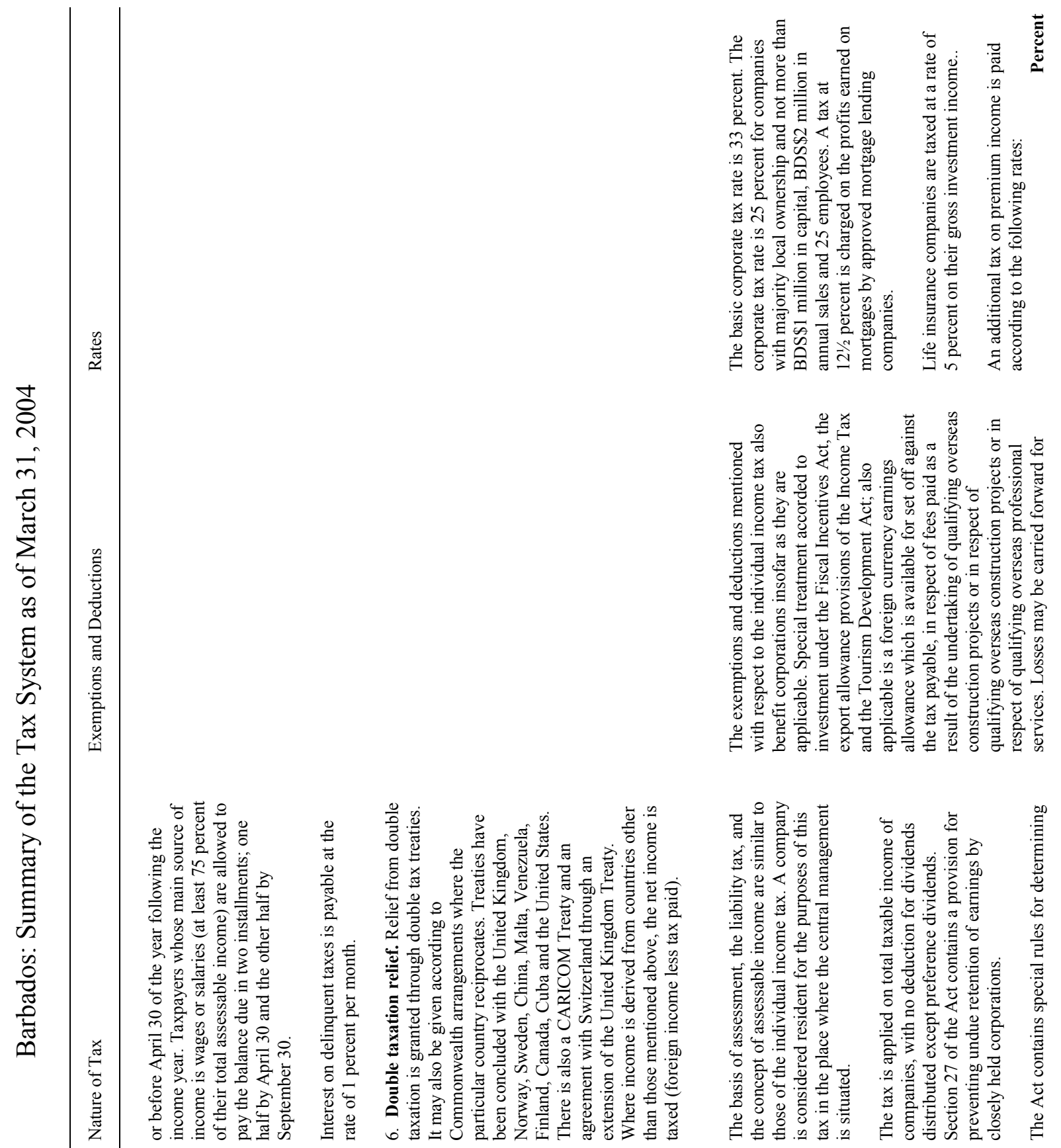




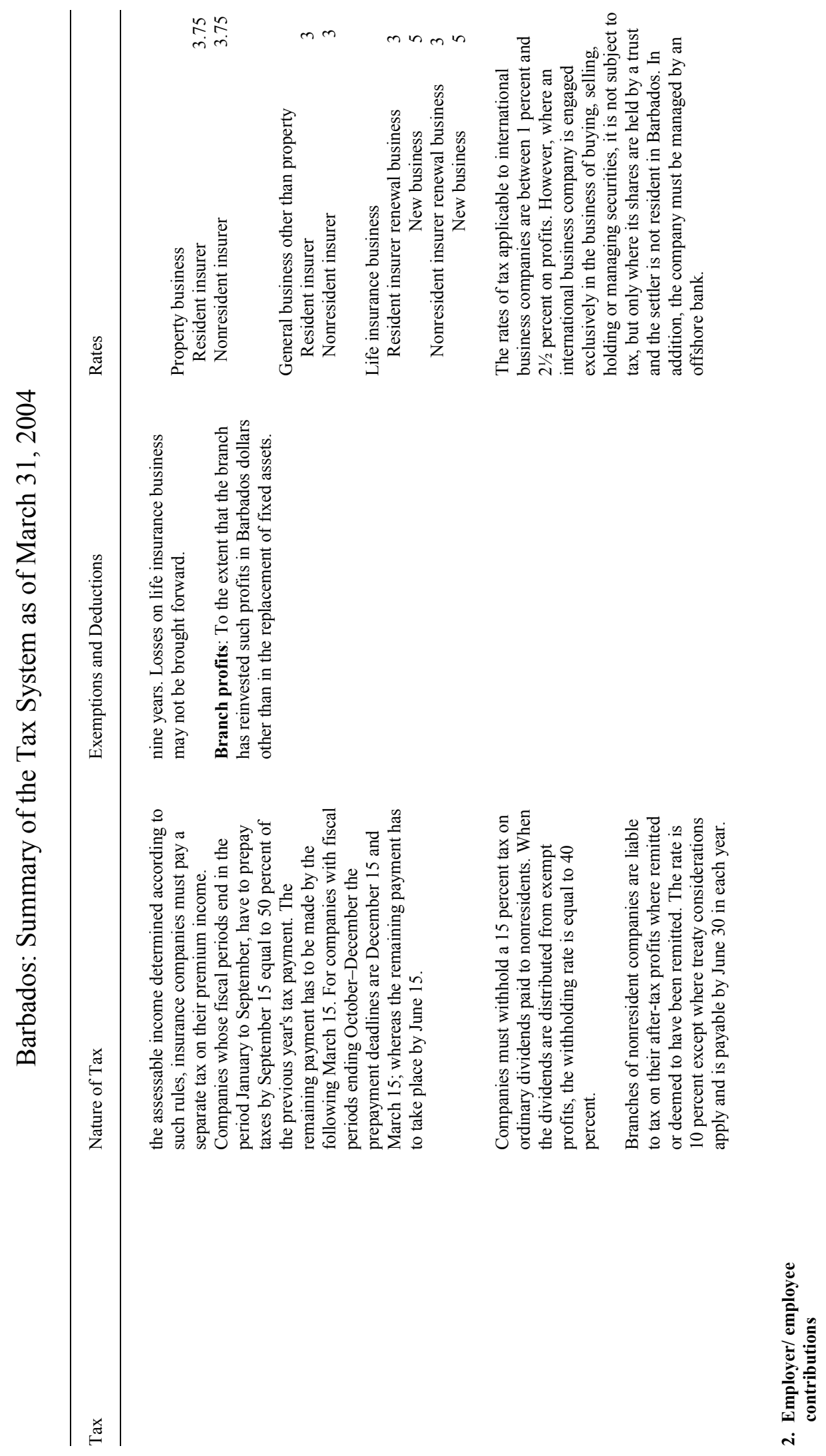



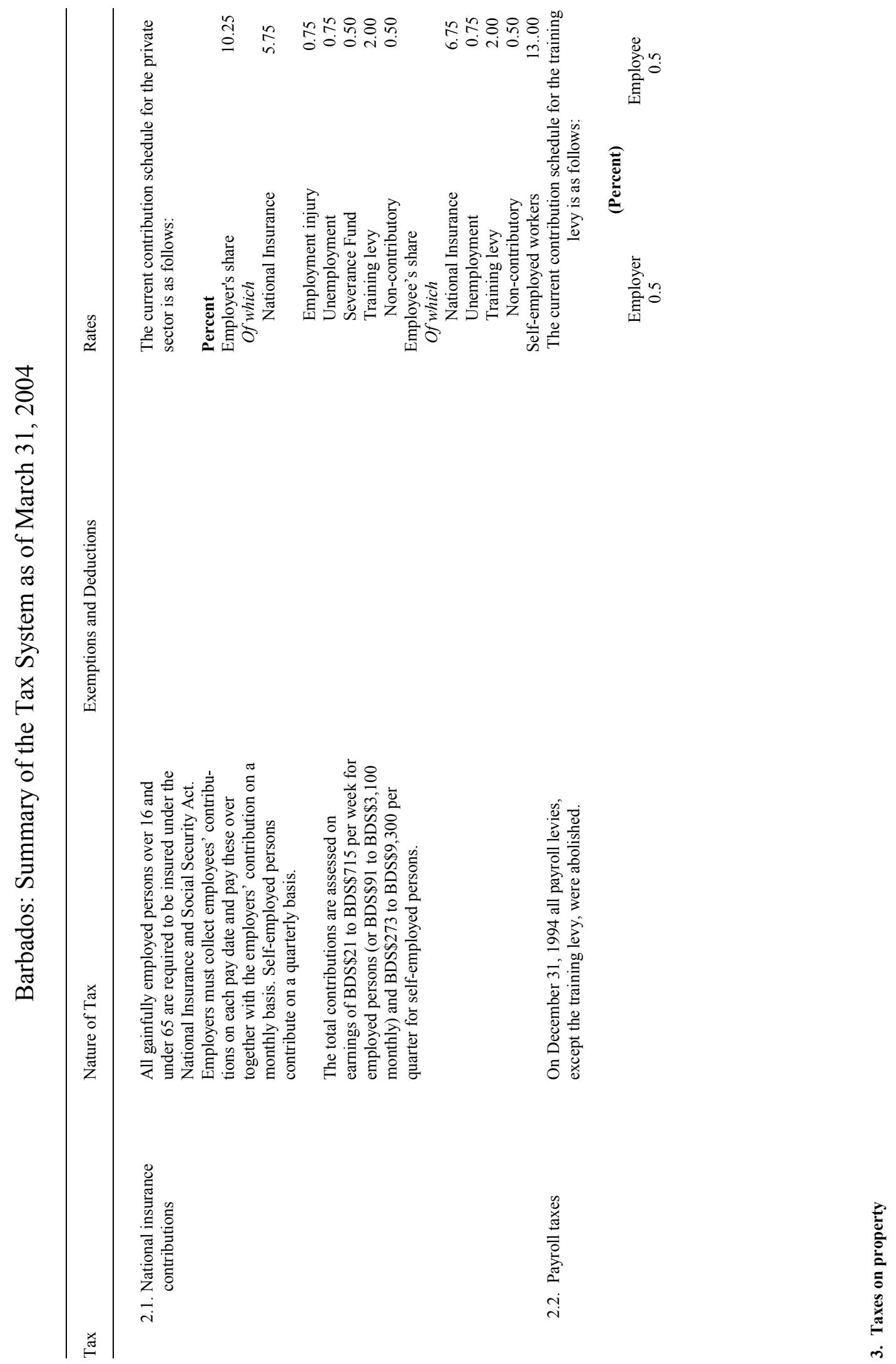


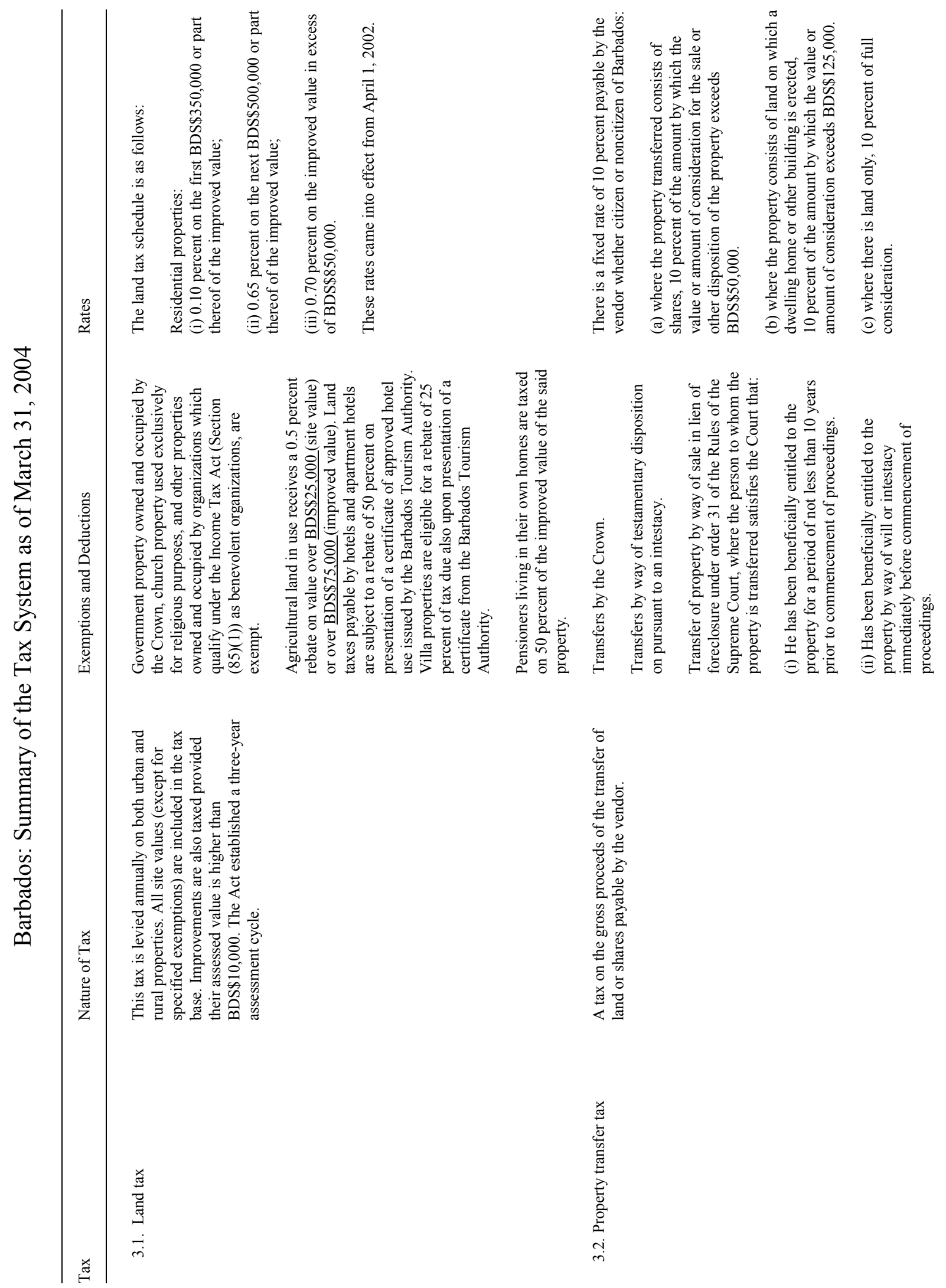




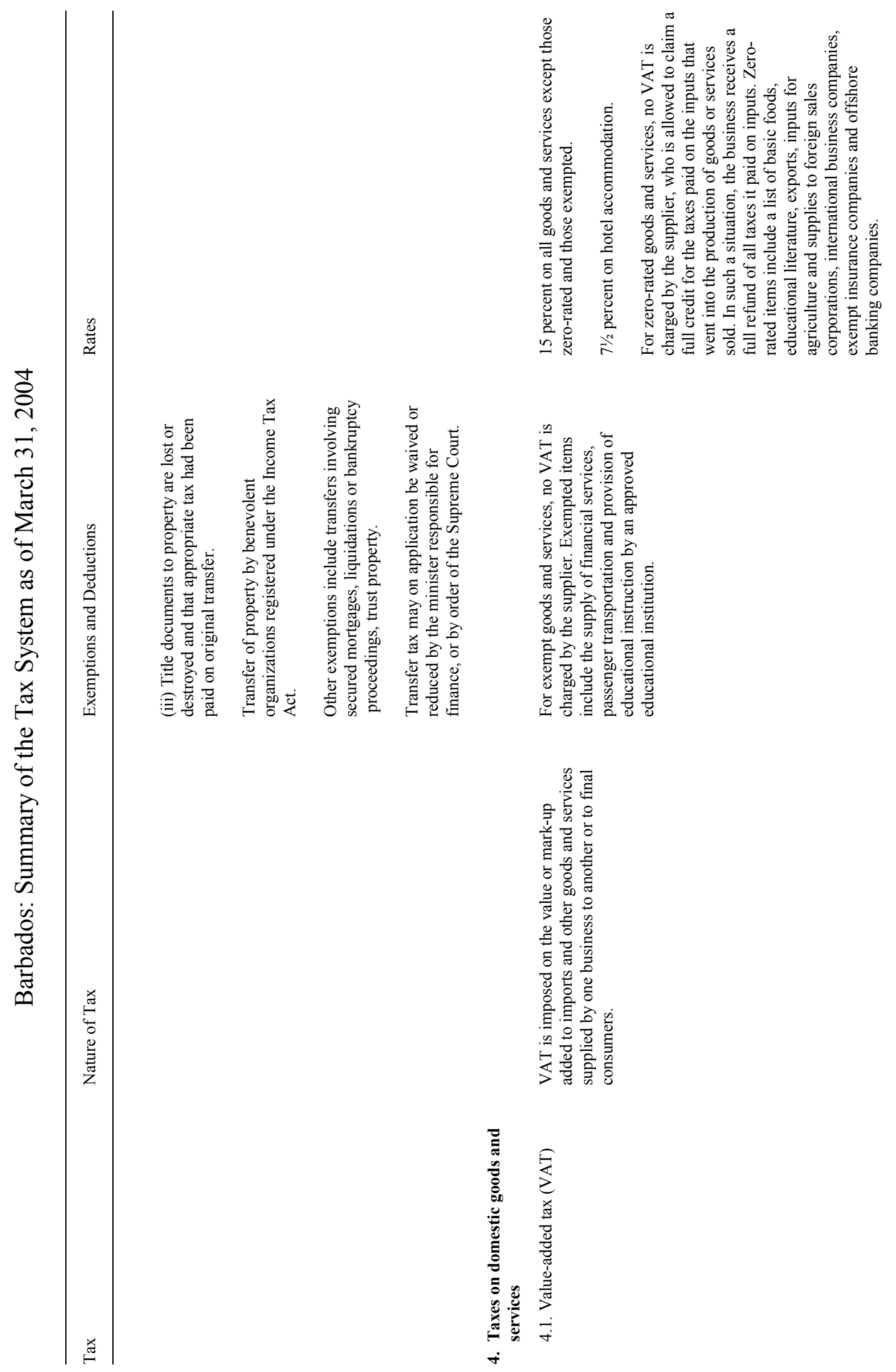




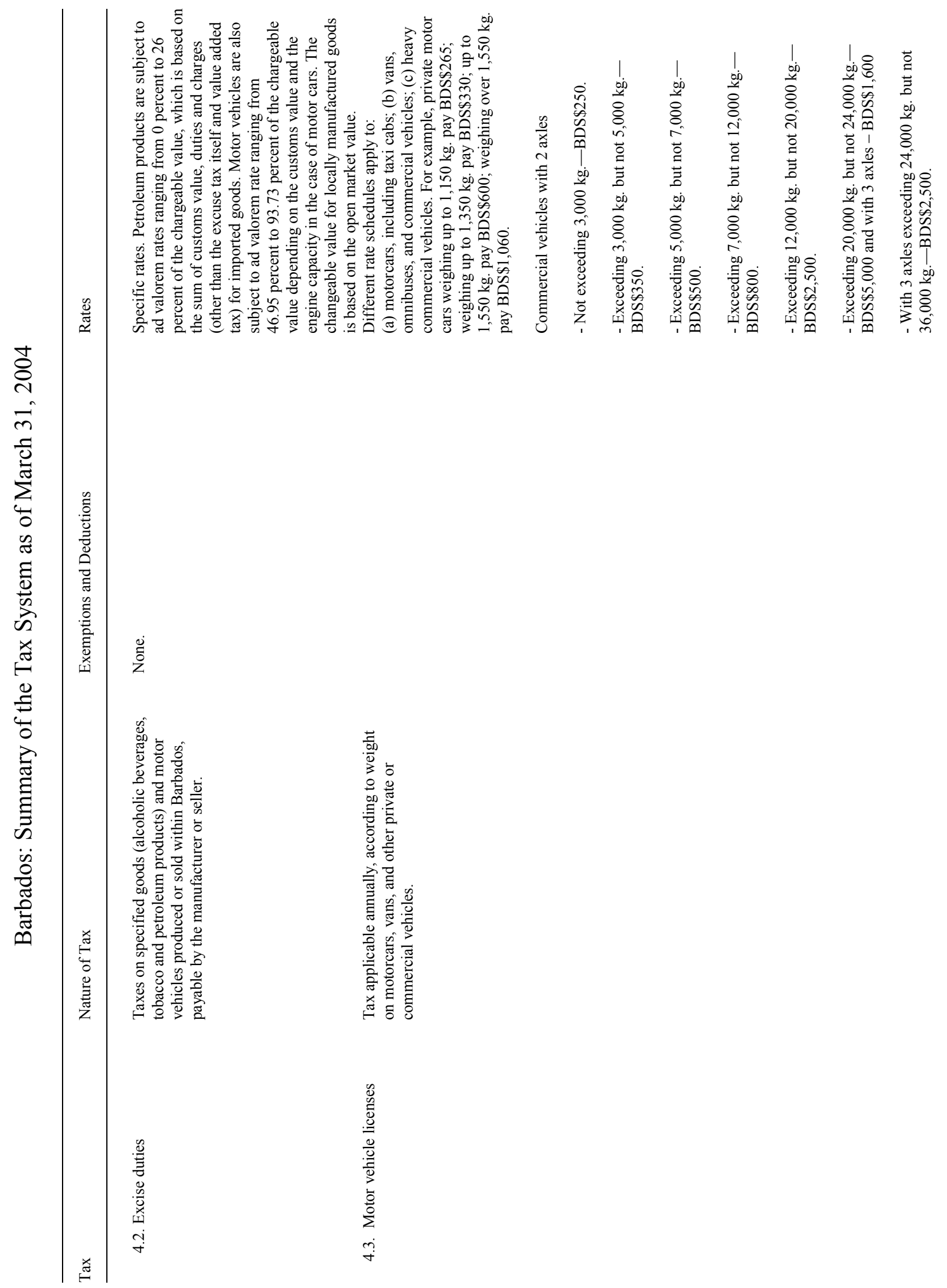



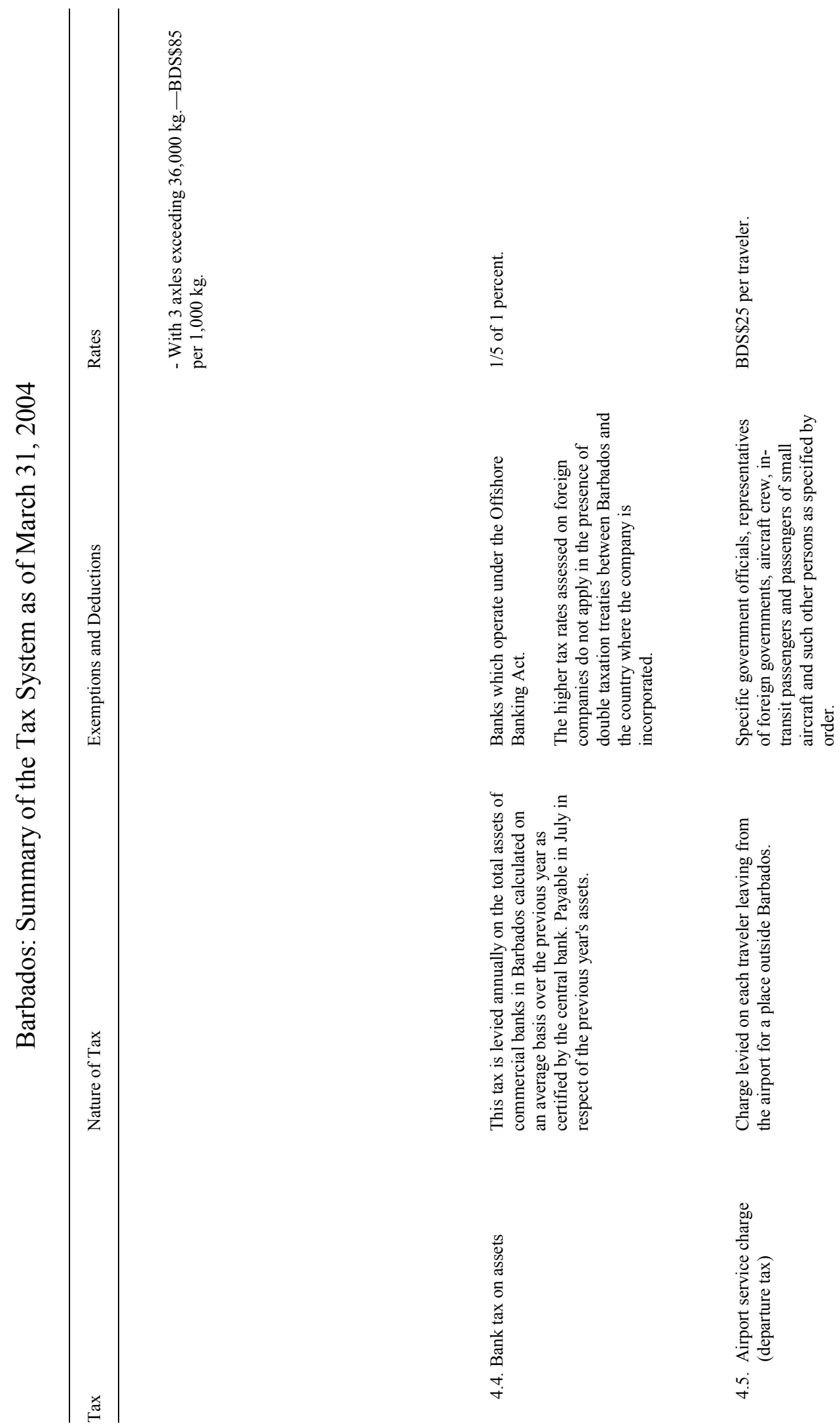


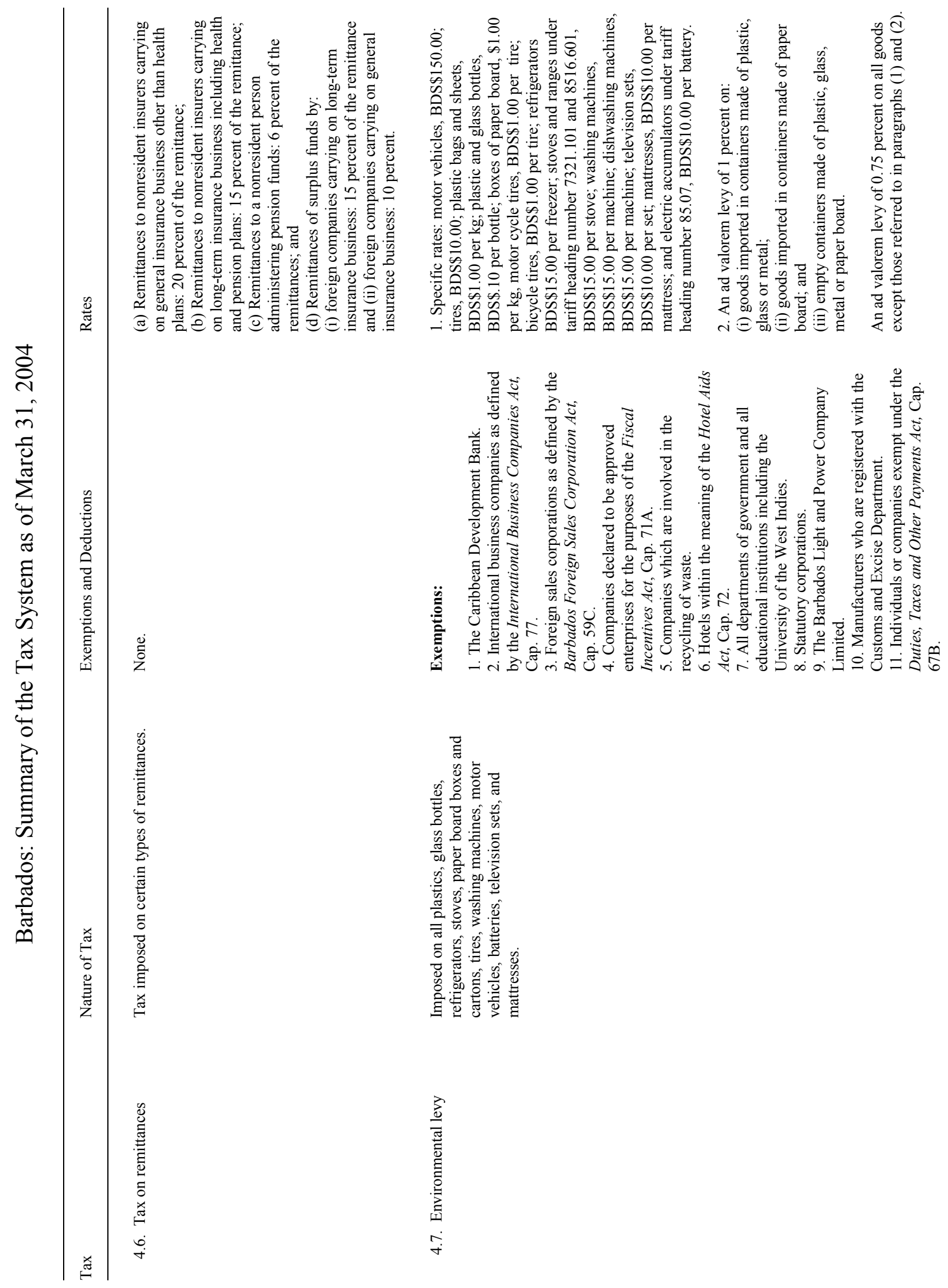




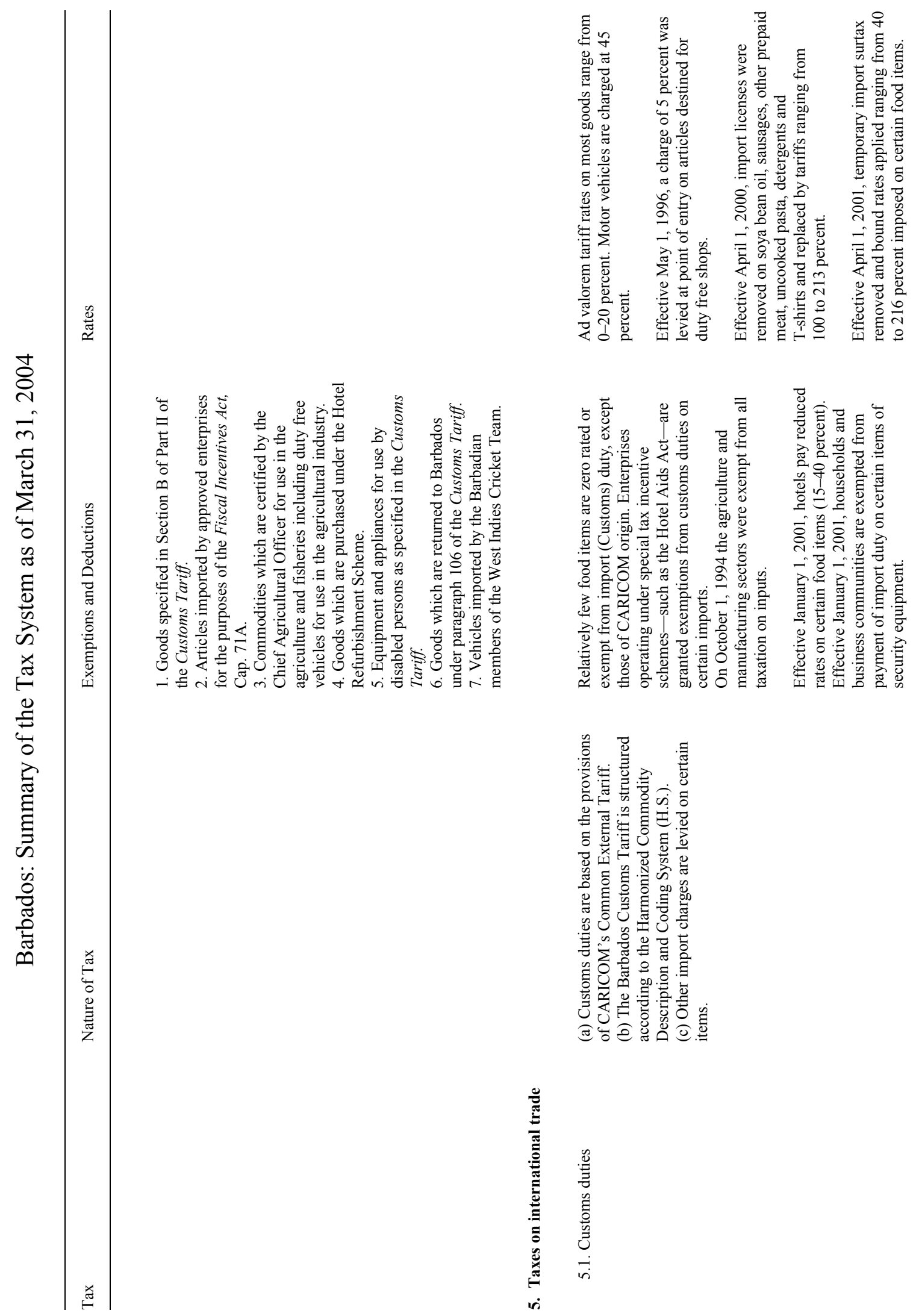

